\title{
Pulsed production of cold protonium in Penning traps
}

\author{
Sebastian Gerber* and Michael Doser \\ CERN, European Laboratory for Particle Physics, 1211 Geneva, Switzerland \\ Daniel Comparat \\ Laboratoire Aimé Cotton, CNRS, Université Paris-Sud, ENS Cachan, Bât. 505, 91405 Orsay, France
}

(Received 8 July 2019; published 16 December 2019)

\begin{abstract}
Precision comparison experiments on bound states of matter and antimatter rely on the production of corresponding systems at low temperatures and in sufficient numbers. In this paper we propose a scheme for the pulsed production of highly excited protonium $(\mathrm{Pn})$ in a Penning-Malmberg trap at low kinetic energies of tens of meV. The scheme relies on the resonant-charge-exchange reaction $\mathrm{H}^{*}+\bar{p} \rightarrow \mathrm{Pn}^{*}+e^{-}$where Rydberg excited hydrogen and antiprotons $(\bar{p})$ interact to form $\mathrm{Pn}^{*}$. The reagent $\mathrm{H}(n=30, l=2)$ is created from laser photodetached and excited hydrogen anions $\left(\mathrm{H}^{-}\right)$, which are initially trapped and mixed in a plasma together with electrons and antiprotons at low kinetic energies. We discuss a three-step pulsed laser excitation using rate equations. A semiclassical Monte Carlo approach leads to a formation rate of $10^{5}$ Pn per $20 \mathrm{~s}$ when assuming a production temperature of $100 \mathrm{~K}$. The formed $\mathrm{Pn}$ are internally excited in states with average principal quantum number $\langle n\rangle \approx 1200$ having lifetimes that can reach seconds. The proposed scheme is therefore particularly interesting for experiments aiming at the study of cold antimatter and purely baryonic systems for precision experiments (charge neutrality, gravity, spectroscopy), as performed at the antiproton decelerator facility at CERN.
\end{abstract}

DOI: $10.1103 /$ PhysRevA.100.063418

\section{INTRODUCTION}

Protonium (Pn) consists of the Coulomb bound state of a proton $(p)$ and an antiproton $(\bar{p})$. In comparison to hydrogen $(\mathrm{H})$, the internal energies and intrinsic distances of Pn approximately scale as the ratio of the reduced masses $\mu^{(P n)} / \mu^{(H)}=914$, yielding a ground-state Bohr radius of $57 \mathrm{fm}$. Due to the wave-function overlap of the onium pair, Pn exhibits a finite lifetime before annihilation of one or more quark-antiquark pairs from $p / \bar{p}$, which modifies the properties predominantly of the deeply bound states and of $s$ or $p$ states $[1,2]$. For example, the annihilation width from the $2 p$ state is found to be larger than the radiative width of $0.4 \mathrm{meV}$ with $97.4 \%$ of the total annihilations occurring from the $2 p$ state and only $0.8 \%$ from the $1 s$ state after emission of a $9 \mathrm{keV} \mathrm{x}$ ray [3].

Studies of Pn have been mainly carried out at CERN at the low-energy antiproton ring (LEAR) experiments Obelix and Asterix and at the PS207 experiment [3-5], where Pn has been produced via resonant-charge-exchange of $\bar{p}$ with liquid and gaseous hydrogen. This production method was initially discussed using $\bar{p}$ in Ref. [6]. Through occasional interactions between $\bar{p}$ 's and trace amounts of $\mathrm{H}_{2}^{+}$present in

\footnotetext{
*sebastian.gerber@cern.ch

Published by the American Physical Society under the terms of the Creative Commons Attribution 4.0 International license. Further distribution of this work must maintain attribution to the author(s) and the published article's title, journal citation, and DOI.
}

the experiment's vacuum, Pn was also produced randomly and quasicontinuously at the Athena experiment at the antiproton decelerator (AD) in a Penning trap [7]. While at Athena about 100 Pn per experimental cycle of 3 min were produced, in the case of the LEAR experiments a Pn flux of about $10^{5} \mathrm{~s}^{-1}$ was achieved, with Pn energies in both cases in the $\mathrm{eV}$ range. For these targets using hydrogen molecules in their electronic ground states, due to energy conservation of the constituents, Pn was formed at similar sizes as $\mathrm{H}_{2}$, resulting in initial principal quantum numbers of about $20-40$ and a mean lifetime before annihilation of about $\mu \mathrm{s}[8,9]$. Photons emitted during the cascade of Pn to deeper bound states before annihilation could be observed and the Pn formation processes studied [5]. For the Balmer and Lyman series the hadronic level shifts were measured using a Röntgen spectrometer with a resolution of up to tens of meV [3]. In general, due to the size of $\mathrm{Pn}$ in the fm range, spectroscopic studies directly probe the residual strong force of the Yukawa potential at thresholds.

Pulsed production of Pn would open up the possibility of laser-manipulation of the formed protonium atoms, allowing one to extend the range of experimentally reachable binding energies and thus perform spectroscopic studies on Rydberg transitions of Pn. Due to Doppler line broadening, for such spectroscopic studies it would be beneficial to produce Pn with sub-eV energies. Further, the possibility to produce Pn at these low energies would enable diffraction studies in matter interferometers, e.g., in a Talbot-Lau device [10,11], allowing precision measurements of the charge neutrality between $p$ and $\bar{p}$ or measurements of the gravitational interaction of antimatter with a purely baryonic system. Due to the magnetic moment of the $\bar{p}$ of $\mu_{B} / \mu_{p}=1521$, interferometric 
measurements of Pn would be more robust against magnetic field gradients that can occur within Penning traps, compared to measurements on antihydrogen $(\overline{\mathrm{H}})$. Similarly, precision spectroscopy of transitions between Pn* Rydberg states gives access to the (anti)protonic Rydberg constant in a fully calculable two-body system [9]. Furthermore, extending the Pn production to cold antiprotonic deuterium would allow precision measurements with distinct sensitivities to Lorentz and CPT violation, where the higher intrinsic nucleon momentum of the deuteron enhances Lorentz violation signals $[12,13]$. Finally, because of the absence of density-dependent Stark quenching of excited protonium states, a very large fraction of the formed protonium reaches deeply bound states. A protonium source at rest in vacuum could thus also be used to revisit searches for putative subthreshold dibaryonic [14-16] or hexaquark states [17] with higher sensitivity than in the past by measuring gamma emissions in the range of $0-100 \mathrm{MeV}$ in coincidence with annihilation from $\mathrm{x}$-ray-tagged $\mathrm{Pn}(1 s)$ or $\operatorname{Pn}(2 p)$ states.

In this article we theoretically investigate a pulsed production scheme of Pn with energies of tens of $\mathrm{meV}$ and with highly excited internal states in a Penning-Malmberg trap. We propose to increase the Pn yield by several orders of magnitude compared to the previous Penning trap experiment (Athena [7]) in a three-step process: (1) trapping, electron cooling, and plasma preparation of mixed $\bar{p}$ and $\mathrm{H}^{-}$at cryogenic temperatures, (2) pulsed photodetachment of $\mathrm{H}^{-}$and laser excitation via $\mathrm{H}(2 p)$ to Rydberg states, resulting in (3) formation of Pn from the resonant-charge-exchange reaction

$$
\mathrm{H}(n, l)+\bar{p} \rightarrow \operatorname{Pn}\left(n^{\prime}, l^{\prime}\right)+e^{-} .
$$

This then produces highly excited $\operatorname{Pn}\left(n^{\prime}, l^{\prime}\right)$ states with radiative lifetimes $>10 \mathrm{~ms}$ for $l>5$. This long lifetime is essential for future spectroscopic and gravitational measurements attempts of Pn. To test the proposed scheme over a range of experimental parameters we used a Monte Carlo method to simulate the expected Pn yield and position distribution where the laser-atom interaction is described with a semiclassical rate equation calculation.

\section{Pn PRODUCTION SCHEME}

The proposed experimental setup using a PenningMalmberg trap is sketched in Fig. 1. Initially, $\mathrm{H}^{-}$can be generated from a suitable source, e.g., as presented in Ref. [18], to form a flux of up to $0.1 \mathrm{~mA} \mathrm{~cm}-2$ at beam energies of few keV. Part of the anion beam can then be directed into a cryogenic trap of $B=5 \mathrm{~T}$ and confined by pulsing the axial electric field electrodes. Other magnetic field values could obviously be chosen, but we use this convenient one to illustrate our purpose (mainly because high fields lead to fast electron cooling and lower magnetron motion).

The $\mathrm{H}^{-}$can be overlapped with previously loaded $e^{-}$ and electron cooled for a duration of about $1 \mathrm{~min}$ to reach temperatures in the vicinity of the trap's environment [19]. The $\bar{p}$ delivered by the $\mathrm{AD}$ at intervals of about $100 \mathrm{~s}$ can be caught similarly. The AD upgraded to the ELENA antiproton ring, described in Ref. [20], is estimated to allow for the trapping of about a factor 30 more $\bar{p}$ than with the current $\mathrm{AD}$ facility, thus reaching intensities of $\approx 10^{7} \bar{p}$ per shot. The $\bar{p}$

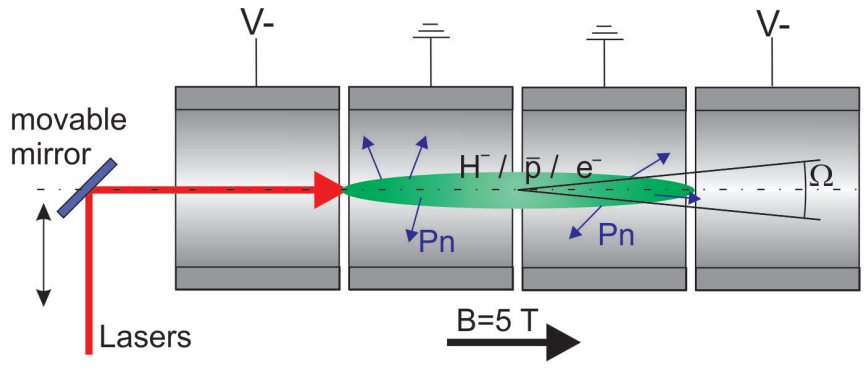

FIG. 1. Sketch of the experimental setup. In a PenningMalmberg trap a plasma of $e^{-}, \mathrm{H}^{-}$, and $\bar{p}$ is confined radially by a $5 \mathrm{~T}$ magnetic field and axially by an electric field. The plasma is addressed axially by three pulsed lasers, which photodetach $\mathrm{H}^{-}$at $1064 \mathrm{~nm}$ and excite the resulting $\mathrm{H}$ on the Lyman-alpha $\mathrm{H}(1 s)$ to $\mathrm{H}(2 p)$ transition at $121 \mathrm{~nm}$ and on the $\mathrm{H}(2 p)$ to $\mathrm{H}(30 d)$ transition at $366 \mathrm{~nm}$. At that time Pn production starts via resonant-chargeexchange of $\mathrm{H}$ and $\bar{p}$, and the Pn are emitted according to their angular velocity distributions. Part of the Pn can escape in forward direction into a solid angle of $\Omega$.

bunch can be electron cooled in similar manner as the $\mathrm{H}^{-}$, and energies down to tens of $\mathrm{meV}$ are regularly achieved at $\mathrm{AD}$ experiments [21-24]. For this paper we choose a plasma temperature of $100 \mathrm{~K}$ as a convenient and conservative choice to illustrate the proposed scheme. At this stage, the two plasmas can be brought to overlap by electric field manipulation on the electrodes. Using a rotating wall drive by applying a dipole field to a four-segmented electrode, the mixed plasma can be compressed and prepared at adjustable densities resulting in plasma radii $r_{p}$ in the $\mathrm{mm}$ range and in lengths $l_{p}$ up to hundreds of $\mathrm{mm}$, depending on the participating particle numbers. After thermalizing again, part of the $e^{-}$can be removed by a series of short rf pulses applied to the axial confining electrode, described in Ref. [25]. Assuming heating rates due to residual gas pressure and trap inhomogeneities of about $10 \mathrm{mK} \mathrm{s}^{-1}$ [26], an approximate number ratio consisting of $\sim 10 \% e^{-}$ensures being able to maintain the temperature [19].

Collisions between $\bar{p}$ and $\mathrm{H}^{-}$or $\mathrm{H}$ can cause resonantcharge-exchange formation to Pn, where the formation cross sections are mainly determined by the collision kinetic energy $E_{k}$ of the $\bar{p}$ with the matter atoms and by the quantum state the atoms reside in. At $E_{k}<2.7 \mathrm{eV}$, which is the case for the mixed plasma after electron cooling, the formation cross section of $\mathrm{Pn}$ via $\mathrm{H}^{-}+\bar{p} \rightarrow \mathrm{Pn}+2 e^{-}$can be neglected due to the Coulomb repulsion of the constituents [8]. Thus, formation of Pn is greatly suppressed at the time in the sequence when $\bar{p}$ and $\mathrm{H}^{-}$are merged to one plasma.

For collision with ground-state atoms $\mathrm{H}(1 s)$, the electric field $(E$ ) produced by $\bar{p}$ induces a dipole moment $d \sim \alpha E$ on $\mathrm{H}$ leading to the classical Langevin cross section for the formation process of Eq. (1) as $\sigma_{L}=\pi \sqrt{2 e^{2} \alpha^{(\mathrm{H})} / E_{k}}$ with $\alpha$ the $\mathrm{H}$ polarizability [27-29]. The collision with $\mathrm{H}(1 s)$ has been studied over a wide energy range of $\mu \mathrm{eV}$ to $\mathrm{keV}$ by comparing analytical to classical trajectory Monte Carlo (CTMC) models in Ref. [30], by using CTMC pseudopotential models in Ref. [31], and by using adiabatic methods in Refs. [32,33]. For relative energies of $<1.5 \mathrm{eV}$ the process can be approximated 
in an adiabatic formation picture [34]. However, for collisions with $\mathrm{H}(n)$ with states $n>1$ the situation is quite different because the level degeneracy leads to a situation in which the dipole $d$ has a permanent component (linear Stark shift). This modifies the scaling of the incoming kinetic energy $E_{k}$ in the cross section to $\propto E_{k}^{-1}$ for low $E_{k}$. This has been recently studied in CTMC simulations of $\bar{p}$ collisions with $\mathrm{H}(2 s)$ and $\operatorname{Ps}(2 s)$ in Ref. [35,36], and with $\operatorname{Ps}(n \leqslant 8)$ in Ref. [37], and $\operatorname{Pn}(n \leqslant 50)$ in Ref. [38].

When the de Broglie wavelength of the atom is comparable to its size, quantum suppression can lead to a varying of the cross section with $n^{2}$ away from its geometrical $n^{4}$ dependence [37]. But for $n \geqslant 10$ this effect would become relevant only at about $E_{k}<30 \mu \mathrm{eV}$. These results underline that for our considered energy and $\mathrm{H}(n)$ excitation range the formation cross section, $\sigma_{P n}$, scales approximately with $n^{4}$ and $k_{v}^{2}=\left(v_{e} / v_{c m}\right)^{2}$, where $k_{v}$ is the ratio between the electron velocity $v_{e}$ in the $\mathrm{H}$ atom and the center of mass velocity $v_{c m}$ between the $\mathrm{H}$ and the $\bar{p}$, yielding to $\sigma_{\mathrm{Pn}} / n^{4} \propto 1 /\left(n^{2} E_{k}\right)$ in a.u.

Although an exact solution for $\mathrm{H}(n>2)$ (and Rydberg $\mathrm{H}$ ) collisions with $\bar{p}$ would require more in-depth studies, we will employ an approximation from a fitting function, similarly as discussed in Refs. [38-41], to the CTMC results for $\mathrm{H}(n=2)$ from Fig. 6 in Ref. [36] as

$$
\begin{aligned}
\sigma_{P n}\left(n, E_{k}\right) & =n^{4}\left(c 1 k_{v}^{2}+c 2\right), \\
k_{v} & =\frac{v_{e}}{v} c m=\frac{e^{2}}{4 \pi \epsilon_{0} n \hbar} \sqrt{\frac{m_{p}}{2 E_{k}}}, \\
c 1 & =1.49 \times 10^{-24} \mathrm{~m}^{-2}, \\
c 2 & =1.76 \times 10^{-20} \mathrm{~m}^{-2} .
\end{aligned}
$$

Figure 2(a) shows the data points from that figure together with the fit for $\mathrm{H}(n=2)$.

Our formation process [see Eq. (1)] is very similar to Ps $+\bar{p} \rightarrow \overline{\mathrm{H}}+e^{-}$leading to the formation of $\overline{\mathrm{H}}$ through resonant charge exchange of $\bar{p}$ with Ps. In studies of $\operatorname{Ps}(n)$ collisions the scaling of Eq. (2) with $n$ and $E_{k}$ was found to be consistent with CTMC results for $2 \leqslant n \leqslant 50$ in Ref. [38] and for $\operatorname{Ps}(n=2)$ and $\operatorname{Ps}(n=3)$ in Ref. [41]. We can thus use a similar description for the final Pn state distribution as was studied for $\overline{\mathrm{H}}$ formation in Ref. [42]. The Pn states are then expected to be almost fully equipopulated in angular momentum so with all $l_{\mathrm{Pn}}$ states populated and with $2 l_{\mathrm{Pn}}+1$ degeneracy until circular states $\left(l_{\mathrm{Pn}}=n_{\mathrm{Pn}}-1\right)$. As discussed for $\mathrm{H}(n=1)$ in Ref. [34], generally with higher $E_{k}$ the $l_{\mathrm{Pn}}$ distribution becomes broader and can approach values of the orbiting angular momentum $l_{\mathrm{Pn}, o r b} \approx\left[8 \alpha^{(\mathrm{Pn})}\left(\mu^{(\mathrm{Pn})}\right)^{2} E_{k}\right]^{1 / 4}$ (in atomic units). Here $\mu^{(\mathrm{Pn})}$ is the reduced mass of Pn, which will be outlined in the Appendix. The $n$ distribution is mainly determined by energy conservation $E^{(\mathrm{Pn})} \approx\left(\mu^{(\mathrm{Pn})} / \mu^{(\mathrm{H})}\right) E^{(\mathrm{H})}+E_{k}$, with the $\mathrm{H}$ binding energy $E^{(\mathrm{H})}=\mathrm{Ry}^{(\mathrm{H})} / n_{\mathrm{H}}^{2}$ and $\mathrm{Ry}^{(\mathrm{H})} \approx 13.6 \mathrm{eV}$. The quantum state of $\mathrm{Pn}$ then scales with the one of the $\mathrm{H}$ atom $n_{\mathrm{Pn}}=n_{\mathrm{H}} \sqrt{\mu^{(\mathrm{Pn})} / \mu^{(\mathrm{H})}} \approx 30.2 n_{\mathrm{H}}$. The energy conservation we wrote was only approximate because the kinetic energy of the ejected electron is not included. However, we know that the Pn must have binding energies larger than $E^{(\mathrm{H})}-E_{k}$ since the electron from the $\mathrm{H}$ has to be ejected with positive energy. Thus, at $E_{k}=E^{(\mathrm{H})}$ there is a cutoff for the formation
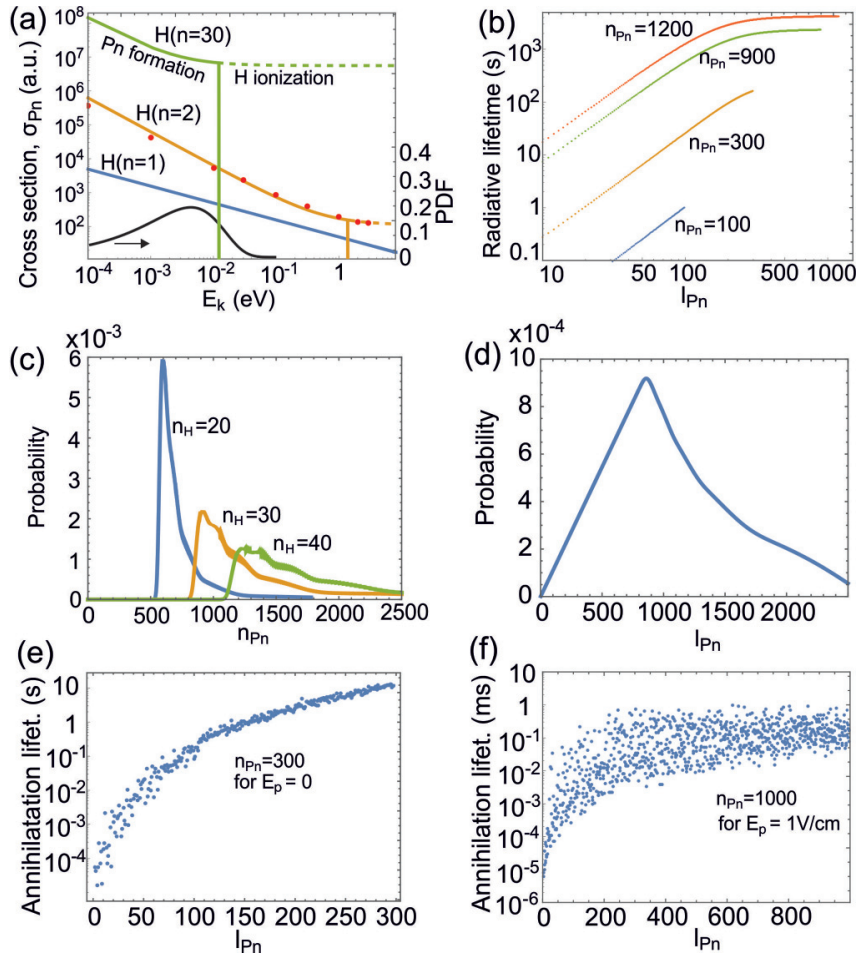

FIG. 2. (a) Formation cross section, versus the collision energy, to produce Pn from $\bar{p}$ : with $\mathrm{H}(n=1)$ using the Langevin cross section, with $\mathrm{H}(n=2)$ and $\mathrm{H}(n=30)$ using Eq. (2). The right axis also indicates the probability distribution function (PDF) for a Maxwellian at $100 \mathrm{~K}$. (b) Radiative lifetime of $100 \leqslant n_{\mathrm{Pn}} \leqslant 1200$ for different $l_{\mathrm{Pn}}$ states including black body radiation at $10 \mathrm{~K}$. (c) $n_{\mathrm{Pn}}$ distribution resulting from $\bar{p}$ capture by either $\mathrm{H}(n=20)$ with $\left\langle n_{\mathrm{Pn}}\right\rangle=$ $735, \mathrm{H}(n=30)$ with $\left\langle n_{\mathrm{Pn}}\right\rangle=1288$, or $\mathrm{H}(n=40)$ with $\left\langle n_{\mathrm{Pn}}\right\rangle=1825$. (d) $l_{\mathrm{Pn}}$ distribution corresponding to $\mathrm{Pn}$ formation from $\mathrm{H}(n=30)$. (e) Time before annihilation occurring in a field-free environment and at $10 \mathrm{~K}$ plotted for initially $\operatorname{Pn}(n=300, l)$. Similarly, panel (f) shows the annihilation time for initial $\operatorname{Pn}(n=1000, l)$ and different $l_{\mathrm{Pn}}$ for a perturbation electric field of $\mathrm{E}_{p}=1 \mathrm{~V} / \mathrm{cm}$.

cross section above which ionization of $\mathrm{H}$ dominates [43,44]. A full quantum mechanical treatment in Ref. [45] for $\mathrm{H}(1 s)$ confirms the steep formation rate drop with a small formation probability continuing until $E_{k} \approx 1.2 E^{(\mathrm{H})}$. In the adiabatic picture used here, this translates into integer solutions of $n_{\text {Pn,cutoff }}=\sqrt{\mu^{(\mathrm{Pn})} \mathrm{Ry}^{(\mathrm{H})} /\left[m_{e}\left(E^{(\mathrm{H})}-E_{k}\right)\right]}[44,46]$. There is no lower bound of $n_{\mathrm{Pn}}$ imposed, but because this would require large relative kinetic energies of $\mathrm{Pn}$ and of the released $e^{-}$, which are dynamically improbable, the $n$ distribution is shifted to larger values [34].

Similarly as for the discussion of the cross section in Eq. (2), because no results exist for $\mathrm{H}(n)$ collisions for arbitrary $\mathrm{n}$ values, for the $\mathrm{P}(n, l)$ distribution we use results for $\mathrm{H}(n=1)$ and expand the energy scales to higher $\mathrm{H}(n)$ states. Here we use results from Table 2 in Ref. [31], as analytical fits to CTMC simulations. Figure 2(c) then plots the expected $n_{\mathrm{Pn}}$ distribution for Pn formation from $\mathrm{H}(n=30)$ at $T_{p}=$ $100 \mathrm{~K}$, and in comparison from $\mathrm{H}(n=20)$ and $\mathrm{H}(n=40)$, for energies integrated until $E^{(\mathrm{H})}$. The corresponding expected $l_{P n}$ distribution for $n_{\mathrm{H}}=30$ is plotted in Fig. 2(d). 
Assuming a thermalized mixed plasma with a MaxwellBoltzmann energy distribution at temperature $T_{p}$, we define $\eta_{m b, n_{H}}$ as the fraction of collisions from $\bar{p}$ and $\mathrm{H}(n)$ to occur at energies $<E^{(H)}$. Here we will focus on Pn formation from $n_{\mathrm{H}}=30$, which choice we will discuss in more detail in Sec. III, resulting in $\eta_{m b, 30}=0.68$. Figure 2(a) then plots the formation cross sections for the states $\mathrm{H}(n=1), \mathrm{H}(n=2)$, and $\mathrm{H}(n=30)$, where an increase of about four orders of magnitude can be seen when comparing $\mathrm{H}(n=1)$ to $\mathrm{H}(n=$ $30)$. The classical cutoffs at $E^{(\mathrm{H})}$ are shown together with the energy distribution at $100 \mathrm{~K}$, resulting in $\eta_{m b, 30}=0.68$.

Figure 2(b) shows the radiative lifetimes for different $\operatorname{Pn}(n, l)$ states in a cryogenic trap environment at $10 \mathrm{~K}$. Here the black body radiation (BBR) at $10 \mathrm{~K}$ is included, which can cause stimulated transitions and photoionization. For the latter we use the rate $\Gamma_{b b r}=c \int_{\omega_{n l}}^{\infty} \rho_{\omega} \sigma_{\omega} d \omega$, where $\omega_{n l}=1 /\left(2 n^{2}\right)$ is the photoioinization threshold frequency in a.u., $\rho_{\omega}$ is the volume density of the BBR photons given by the Planck distribution, and $\sigma_{\omega}$ is the photoionization cross section given by Eq. (A9). At $10 \mathrm{~K}$ for $\operatorname{Pn}(n<1200)$ the BBR-induced photoionization is then negligible compared to the absorption and stimulated emission rate to neighboring states caused by the BBR. From Fig. 2(b) the radiative lifetime for $n_{\mathrm{Pn}}>900$ reaches $>100 \mathrm{~ms}$ for states $l_{\mathrm{Pn}}>40$.

Taking as an example the initial state $n_{\mathrm{Pn}}=300$, the time until annihilation occurs is then plotted in Fig. 2(e) for different $l_{\mathrm{Pn}}$, where for each $l_{\mathrm{Pn}}$ all $m_{\mathrm{Pn}}$ states are equipopulated. In this plot, each point represents the time required from an initial $\operatorname{Pn}(n, l)$ state to undergo the radiative decay cascade through intermediate $\mathrm{Pn}$ states in $10 \mathrm{~K}$ until the population reaches low $l$ states and annihilation occurs. Each intermediate $\operatorname{Pn}(n, l) \rightarrow \operatorname{Pn}\left(n^{\prime}, l^{\prime}\right)$ transition is calculated from all possible transition probabilities for time steps of $10 \mathrm{~ns}$ using a Metropolis-Hastings algorithm [47,48]. For each $l_{P n}$ state the simulation of the decay cascade is repeated, giving rise to a scattered lifetime plot. The annihilation rates from low $l$ states will be estimated using the Ball-Chew model and scale as $n^{-3}[49,50]$ :

$$
\begin{aligned}
& v_{a}(n s)=5.3 \times 10^{18} \mathrm{n}^{-3} \mathrm{~s}^{-1}, \\
& v_{a}(n p)=4.3 \times 10^{14} \mathrm{n}^{-3} \mathrm{~s}^{-1}, \\
& v_{a}(n d)=10^{10} \mathrm{n}^{-3} \mathrm{~s}^{-1} .
\end{aligned}
$$

Annihilations thus mainly occur from $s$ or $p$ states, where rates from higher values of $l_{P n}$ such as $d$ states are negligible compared to the spontaneous decay rates. From Fig. 2(e) the lifetimes reach seconds for $l_{P n}>150$.

To estimate an initial Pn yield, the envisaged resonantcharge-exchange reaction from $\bar{p}$ capture of $\mathrm{H}(30 d)$ relies on the formation cross section $\sigma_{\mathrm{Pn}}$, the collision energy, and the number of available constituents. The expected number of inelastic collisions can then be estimated for a plasma with homogeneous cylindrical shape of radius $r_{p}$ and length $l_{p}$ using Beer-Lambert's law

$$
N_{\mathrm{col}}=N_{\mathrm{H}}\left[1-\exp \left(-\sigma_{P n} n_{\bar{p}} D\right)\right],
$$

with $N_{P n}=\eta_{m b, 30} N_{\text {col }}$. Here $N_{\mathrm{H}}$ is the number of Rydberg $\mathrm{H}$ atoms interacting with the $\bar{p}$ at a density $n_{\bar{p}}$ and $D$ is the mean distance of any point inside the plasma to the plasma edge. For this simple estimation we use the crude approximation
$D=\min \left(2 r_{p}, l_{p}\right)$. For values of $r_{p}=1 \mathrm{~mm}, l_{p}=100 \mathrm{~mm}$, $n_{\bar{p}}=10^{14} \mathrm{~m}^{-3}, \sigma_{\mathrm{Pn}}=1.4 \times 10^{-13} \mathrm{~m}^{-2}$ [Eq. (2) at $\mathrm{H}(n=30)$ and at kinetic energy corresponding to a plasma temperature of $T=100 \mathrm{~K}$ of $E_{k}=8.6 \mathrm{meV}$ ], and $N_{\mathrm{H}}=10^{6}$ we expect about $N_{\mathrm{Pn}} \approx 3 \times 10^{4}$. In $B=5 \mathrm{~T}$ the energy from plasma rotation of $\bar{p} / \mathrm{H}^{-}$would be $0.3 \mathrm{meV}$, which is smaller than the thermal energy and is neglected for this estimation using Eq. (4). This simple estimation is confirmed by a full Monte Carlo simulation. For doing this we first need to study the laser interaction in more detail.

\section{A. $\mathrm{H}^{-}$excitation to Rydberg $\mathrm{H}$}

To create Rydberg $\mathrm{H}$ from ground-state $\mathrm{H}^{-}$and to calculate the value for the laser excitation efficiency $\eta_{L}$, several laser excitation paths are possible [51]. However, for an accessible experimental realization we concentrate on a scheme using single-photon excitations and a Lyman- $\alpha$ laser. First a pulsed laser at $1064 \mathrm{~nm}$ photodetaches $\mathrm{H}^{-}$from the ground state to the $\mathrm{H}(1 s)$ state, overcoming the binding energy of $\mathrm{BE}=0.7542 \mathrm{eV}$ [52]. The photodetachment cross section is about $\sigma_{1064}=4 \times 10^{-21} \mathrm{~m}^{2}$ [53-55]. Subsequently, a laser at $121 \mathrm{~nm}$ excites the atoms to the $\mathrm{H}(2 p)$ state, from where a third laser at $366 \mathrm{~nm}$ addresses the $\mathrm{H}(2 p) \rightarrow \mathrm{H}(30 d)$ transition.

Figure 3(a) sketches the relevant levels of $\mathrm{H}$ and $\mathrm{H}^{-}$in the Paschen-Back regime at $B=5 \mathrm{~T}$ showing the uncoupled basis $\left|n, l, s, m_{l}, m_{s}\right\rangle$ for each state (with obvious notations for the orbital and spin components), where the level splitting is $\Delta E=\mu_{B} B\left(m_{l}+2 m_{s}\right)$. The splitting caused by a change of $\Delta m_{l}= \pm 1$ or $\Delta m_{s}= \pm 1 / 2$ is then $11.2 \mathrm{GHz}$. In the scheme (see Fig. 1) the lasers illuminate the plasma along the $B$ field and drive $\sigma$ transitions, where we choose a laser polarization of $\sigma^{-}$for the $121 \mathrm{~nm}$ and $\sigma^{+}$for the $366 \mathrm{~nm}$. For the $121 \mathrm{~nm}$ we will use specifications that have been achieved in Ref. [56] of a laser energy of $190 \mathrm{~nJ}$ at a pulse duration of $16 \mathrm{~ns}$, which results in a line width of $\delta v_{L} \approx 27 \mathrm{MHz}$. For the other two lasers we will fix the pulse duration to typical $10 \mathrm{~ns}$ giving line widths of $\approx 44 \mathrm{MHz}$. The $\mathrm{H}$ transitions get Doppler broadened at the plasma temperature, which gives at $T_{p}=100 \mathrm{~K}$ a transition FWHM of $\Delta v_{D, 121}=17.9 \mathrm{GHz}$ and $\Delta v_{D, 366}=5.9 \mathrm{GHz}$. Because this broadening is larger than the hyperfine splittings between $m_{i}= \pm 1 / 2$ states of $\Delta E_{H F}=$ $1.3 \mathrm{GHz}$ for the $\mathrm{H}(1 s)$ and of $55 \mathrm{MHz}$ for the $\mathrm{H}(2 p)$, all $m_{i}$ hyperfine substates are addressed.

From this we see that after the $1064 \mathrm{~nm}$ addresses the singlet $\mathrm{H}^{-}$state, all four $m_{i} \mathrm{H}(1 s)$ hyperfine states get populated. Then, due to Doppler broadening at $100 \mathrm{~K}$ the $121 \mathrm{~nm}$ laser addresses all $\mathrm{H}(1 s)$ substates. To find the overall transfer efficiency, we can calculate the cross sections and the excitation and photoionization rates using standard expressions for the radial wave functions of $\mathrm{H}$ and their overlaps for bound-bound and bound-continuum transitions (see the Appendix). The excitation rates include the Doppler broadening at the plasma temperature. Both the $121 \mathrm{~nm}$ and $366 \mathrm{~nm}$ laser can cause photoionization from the excited states. The photoionization rates for the $121 \mathrm{~nm}$ laser from $\mathrm{H}(2 p), \Gamma_{p i, 2 p}$, is calculated from the sum of the rates coupling to the $l=0$ and $l=2$ continuum states. Similarly, the photoionization rate from $\mathrm{H}(30 d)$ caused by the $121 \mathrm{~nm}$ and the $366 \mathrm{~nm}$ laser as $\Gamma_{p i, 30 d}$ 

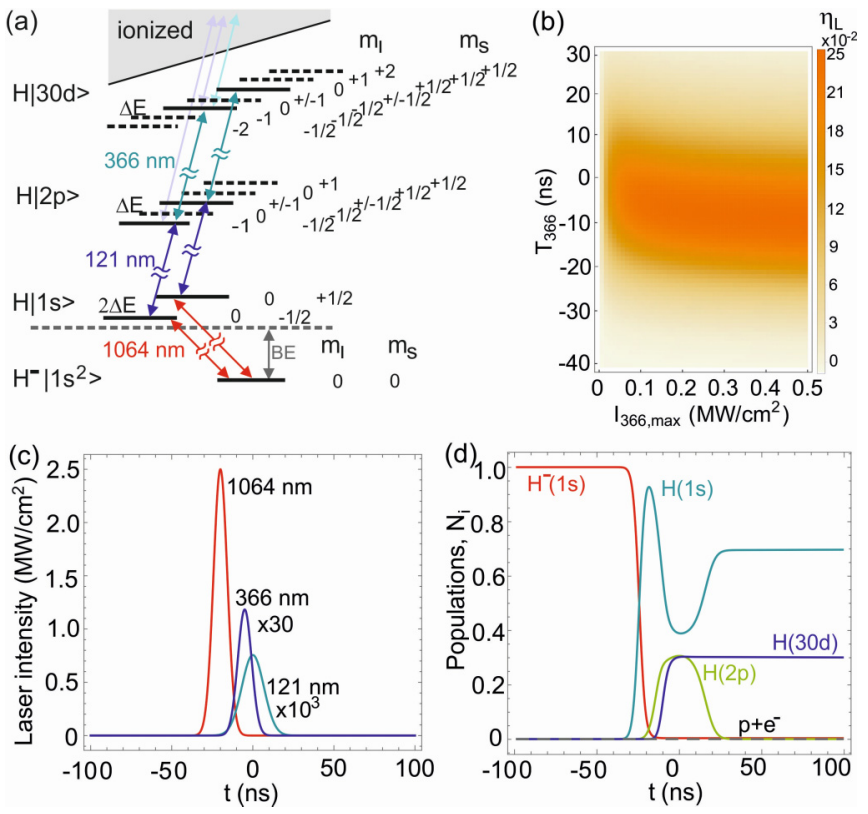

FIG. 3. (a) Level scheme of the relevant states of $\mathrm{H}^{-}$and $\mathrm{H}$ at $5 \mathrm{~T}$. The continuum of $\mathrm{H}$ is marked in gray with the possible ionizations from the excited states. The $m_{l}, m_{s}$ states and $\Delta E$ are labeled and the binding energy $(\mathrm{BE})$ of $\mathrm{H}^{-}$. The three lasers are shown with the $121 \mathrm{~nm}$ and $366 \mathrm{~nm}$ laser driving $\sigma^{-}$and $\sigma^{+}$transitions, respectively. The solid lines represent the addressed states. (b) Excitation efficiency $\eta_{L}$ versus $366 \mathrm{~nm}$ laser parameters for a beam waist of $w_{L}=$ $1 \mathrm{~mm}$ and Gaussian intensity pulse shapes $I_{i}=I_{i, \max } \exp [-4 \ln 2(t-$ $\left.T_{i}\right)^{2} / \tau_{i}^{2}$ ] for $i=1064,121,366$ with peak intensity $I_{i, \max }$, FWHM intensity pulse duration $\tau_{i}$, and pulse delay $T_{i}$ with respect to the $121 \mathrm{~nm} ; I_{1064, \text { max }}=2.5 \mathrm{MW} / \mathrm{cm}^{2}, I_{121, \text { max }}=755 \mathrm{~W} / \mathrm{cm}^{2}, \tau_{1064}=$ $\tau_{366}=10 \mathrm{~ns}, \tau_{121}=16 \mathrm{~ns}$ and $T_{1064}=-20 \mathrm{~ns}$. (c) Laser intensities versus time for $I_{366, \max }=40 \mathrm{~kW} / \mathrm{cm}^{2}$ and $T_{366}=-5 \mathrm{~ns}$. (d) Populations of the bound electronic levels and of dissociated $\mathrm{H}$ from Eq. (5) versus time with an insert showing the $\mathrm{H}(30 d)$ state resulting in $\eta_{L}=0.30$ at $100 \mathrm{~ns}$.

consists of the sums of the individual photoionization rates that couple to the continuum states with $l=1$ and $l=3$. The effect of the laser interaction with the $\mathrm{H}^{-}$and $\mathrm{H}$ atom can then be described as solutions to a set of Einstein rate equations for the time dynamics of the involved atomic populations given by

$$
\begin{aligned}
\dot{N}_{H^{-}}= & -\Gamma_{p d} N_{H^{-}}, \\
\dot{N}_{1 s}= & A_{2 p} N_{2 p}+\Gamma_{p d} N_{H^{-}}-\Gamma_{1 s 2 p}\left(N_{1 s}-N_{2 p}\right), \\
\dot{N}_{2 p}= & -A_{2 p} N_{2 p}+\Gamma_{1 s 2 p}\left(N_{1 s}-N_{2 p}\right) \\
& -\Gamma_{2 p 30 d}\left(N_{2 p}-N_{30 d}\right)-\Gamma_{p i, 2 p} N_{2 p}, \\
\dot{N}_{30 d}= & -A_{30 d} N_{30 d}+\Gamma_{2 p 30 d}\left(N_{2 p}-N_{30 d}\right)-\Gamma_{p i, 30 d} N_{30 d}, \\
\dot{N}_{p i}= & \Gamma_{p i, 2 p} N_{2 p}+\Gamma_{p i, 30 d} N_{30 d} .
\end{aligned}
$$

Here $A_{2 p}=6.3 \times 10^{8} \mathrm{~s}^{-1}$ and $A_{30 d}=2.6 \times 10^{5} \mathrm{~s}^{-1}$ are the Einstein $A$ coefficients from the $\mathrm{H}(2 p)$ and the $\mathrm{H}(30 d)$ state, respectively, and $\Gamma_{p d}$ is the photodetachment rate of $\mathrm{H}^{-}$. Because the decay of $\mathrm{H}(30 d)$ populates many levels with a negligible probability of reaching $\mathrm{H}(2 p)$ within the considered time, we neglected this part. $\Gamma_{1 s 2 p}$ is the $121 \mathrm{~nm}$ laser excitation rate for the transitions $\mathrm{H}(1 s)\left|m_{l}=0\right\rangle \rightarrow$
$\mathrm{H}(2 p)\left|m_{l}=-1\right\rangle$ and $\Gamma_{2 p 30 d}$ is the $366 \mathrm{~nm}$ laser excitation rate for $\mathrm{H}(2 p)\left|m_{l}=-1\right\rangle \rightarrow \mathrm{H}(30 d)\left|m_{l}=0\right\rangle$, because we have used circular polarized light. $N_{p i}$ is the population lost due to photoionized $\mathrm{H}$. We define the population transfer efficiency from $\mathrm{H}^{-}$to $\mathrm{H}(30 d)$ as $\eta_{L}=N_{30 d} / N_{\mathrm{H}^{-}}$at $t=100 \mathrm{~ns}$. This parameter is maximized scanning over the laser intensities and pulse delays; see Fig. 3(b). Figure 3(c) shows the time-dependent populations of the states in Fig. 3(d). Here we find that $\sim 30 \%$ of the $\mathrm{H}^{-}$are excited to $\mathrm{H}(30 d)$ mainly limited by the Lyman- $\alpha$ laser power and spectral width.

\section{B. Pn limitations from crossed fields in Penning traps}

The preceding discussion deals (especially the Pn crosssection formation and $\mathrm{Pn}$ radiative lifetime and annihilation) with Pn in field-free $|n l m\rangle$ state. But the Pn evolves in a complex electric and magnetic field environment. Indeed, formed Pn will be exposed to the trapping magnetic field but also to several electric fields: time-constant electric fields (due to the trapping potential in axial $\mathbf{E}_{a x}$ and radial $\mathbf{E}_{\mathrm{rad}}$ direction) but also time-varying electric fields (from stochastic Coulomb collision with $p, \bar{p}, e^{-}, \mathrm{H}^{-}, \mathrm{H}^{*}$ ) and a motional field $\mathbf{E}_{m}=$ $\mathbf{v} \times \mathbf{B}$. It is beyond the scope of this article to study in detail the effect of the external fields, and we will simply give simple arguments in order to estimate the modification of the internal states and especially concerning the effect on annihilation. The Hamiltonian for Pn in $\mathbf{E}$ and $\mathbf{B}$ fields is expressed as $H=$ $H_{0}+H_{\text {Zeeman }}+H_{\text {Diamagnetic }}+H_{\text {Stark }}$ and is discussed in the Appendix (the formulas are also valid for $\mathrm{H}$ and Ps because of the reduced mass). The Zeeman effect of Pn [see Eq. (A1) in the Appendix [ is very small and occurs only from the nuclear spin. Furthermore, the first-order (orbital) Zeeman effect is zero. We can thus simply regard Pn as affected only by the Stark effect. The Hamiltonian eigenstates are thus the Stark eigenstates (assuming a quantization axis along the electric field): $|n k m\rangle=\sum_{l}|n l m\rangle C_{\frac{n-1}{2} \frac{m+k}{2}, \frac{n-1}{2} \frac{m-k}{2}}^{l m}$ where $C_{j_{1} m_{1}, j_{2} m_{2}}^{j m}$ is the standard Clebsch-Gordan coefficient. This formula can be used to estimate the annihilation lifetime of the Stark states because of the $s$ or $p$ annihilation rate; see Eq. (3).

In a very rough estimation we can assume that the ClebschGordan coefficient is on the order of $1 / n$ and that the Stark eigenstates contain more or less all $l \geqslant m$ quantum numbers. Therefore the annihilation rate for a Stark eigenstate with $m=0$ is roughly given by $\sim v_{a}(n s) n^{-1}$ and for a $m=1$ state by $\sim v_{a}(n p) n^{-1}$. For $m= \pm 2$ the radiative transition rate into $p$ states is larger than the $d$-state annihilation rate. The first net important result is that annihilation dominates from $m=$ $0, \pm 1$ Stark states and that particularly higher $m$ states can be thought to be stable against annihilation.

The Pn are initially formed inside a plasma where the Debye shielding makes the mean (global) electric field to be zero, and the Pn are thus formed in $|n l m\rangle$ states. The key question that arises is what happens during the Pn motion, when Pn will see modification of its environment, for instance, by moving from a zero field region, with $|n l m\rangle$ eigenstates, to a region with presence of an electric field, with $|n k m\rangle$ eigenstates. The frontier is given by comparing the evolution time $\tau$ and the typical energy gap of the Hamiltonian [57-59]. For instance, a Pn will stay in its initial $|n l m\rangle$ level (sudden approximation) if $\tau \ll \frac{\hbar}{\Delta \bar{H}}$ where $\Delta \bar{H}$ is the root mean 
square deviation of the system Hamiltonian averaged over the interval of interest. This can be estimated using the matrix elements for $H_{\text {Stark }}$ (where $z$ is the quantization axis where $m$ is defined) $[27,60,61]$ :

$$
\begin{aligned}
v_{S t, x}(n l m) & =\frac{e}{\hbar}|\langle n l m|\mathbf{E} x| n l-1 m \pm 1\rangle| \\
& =\frac{3}{2} \frac{e a_{0}^{(\mathrm{Pn})}}{\hbar}|\mathrm{E}| n \sqrt{\frac{(l \mp m-1)(l \mp m)\left(n^{2}-l^{2}\right)}{4\left(4 l^{2}-1\right)}} \mathrm{s}^{-1}, \\
v_{S t, z}(n l m) & =\frac{e}{\hbar}|\langle n l m|\mathbf{E} z| n l-1 m\rangle| \\
& =\frac{3}{2} \frac{e a_{0}^{(\mathrm{Pn})}}{\hbar}|\mathrm{E}| n \sqrt{\frac{\left(l^{2}-m^{2}\right)\left(n^{2}-l^{2}\right)}{4 l^{2}-1}} \mathrm{~s}^{-1},
\end{aligned}
$$

where $a_{0}^{(P n)}=914 a_{0}^{(H)}$ is the Bohr radius of Pn. These are the rates at which the $|n l m\rangle$ states are mixed. The detailed theory of the complex evolution of all states under varying electric field is obviously very complex and out of the scope of this article. Therefore we will use a simple shuffling model as developed in Refs. [27,60]. This model will give only a rough order of magnitude for the evolution and takes into account the net effect of back and forth "transitions" between different $\operatorname{Pn}(n, l, m \leftrightarrow n, l-1, m \pm 1)$. The simple picture is thus that an electric field along $z$ causes the Pn to oscillate continually between all degenerate $l$ states with a frequency of $\sim \sum_{l=0}^{l=n-1}\left(v_{S t, z}^{2}\right)^{1 / 2}$ and for an orthogonal field at a rate $\sim \sum_{l=0}^{l=n-1} \sum_{m=-l}^{m=l}\left(v_{S t, x}^{2}\right)^{1 / 2}$. For the case of a perturbation field of $\mathrm{E}_{p}=1 \mathrm{~V} / \mathrm{cm}$, Fig. 2(f) shows the lifetime before annihilation occurs for the case of an initial state $\operatorname{Pn}(n=1000, l)$. Using time steps of $1 \mathrm{~ns}$, the number of Stark transitions for each $l_{\mathrm{Pn}}$ and using equipopulated $m_{\mathrm{Pn}}$ states are calculated from Eq. (6) giving $v_{S t, x} \sim 10^{7} \mathrm{~s}^{-1}$. From Eq. (3) this then leads to a reduction of the annihilation lifetime compared to Fig. 2(e) to about $0.1 \mathrm{~ms}$ for $l_{\mathrm{Pn}}>100$.

As stated previously the effective electric field $\mathbf{E}$ for the Stark effect is the vector sum of many contributions (stochastic, trapping, or motional fields), from whose time dependence the perturbation field can be calculated. This will be done in the next section in a Monte Carlo simulation, but we can first give here some rough estimations.

(1) Concerning the stochastic effect of collisions between Pn and other particles, when a Pn traverses a $\mathrm{H}^{*}$ or a groundstate $\mathrm{H}$ atom it experiences the inner atomic electric field. Similarly the Pn can be subject to the Coulomb field in case of passing close to a $\bar{p}$ or $\mathrm{H}^{-}$. However, for a density $n_{p}=10^{14} \mathrm{~m}^{-3}$, a thermal velocity of the Pn of $v_{t h}=912 \mathrm{~m} / \mathrm{s}$ and $\left\langle n_{\mathrm{Pn}}\right\rangle \sim 1300$, the rate of Coulomb collisions inside the plasma is of the order of $\Gamma_{S t}=n_{p} v_{t h} \pi\left(a_{0}^{(\mathrm{Pn})} n_{\mathrm{Pn}}^{2}\right)^{2} \approx 2.6 \mathrm{~ms}^{-1}$ [61] and is smaller than the travel time through the plasma of order $\mu \mathrm{s}$. This is a first indication that the collisions are probably negligible. It is confirmed by the fact that the typical electric field created by the particle that Pn will encounter will be on the order of $\sim 1 \mathrm{~V} / \mathrm{cm}$ (because this is created by an elementary charge at $4 \mu \mathrm{m}$, which is the typical interparticle distance). And as seen previously this field is not enough to reduce significantly the annihilation lifetime (except for very low $l$ values). Finally this field is just enough to mix the $n \sim 1300$ manifold with the $n+1$ ones (Inglis-Teller fields), but because only a few collisions will arise during the Pn travel, this will probably have a small effect. In conclusion, at first glance, unlike is the case for liquid and gaseous hydrogen targets, the collisions do not seem to play a big role, and we will neglect them here.

(2) For the motion under the trapping field, we will assume $z$ along the $B$ field, and so the axial field does not modify the $m$ values and so does not produce any extra annihilation; see Eq. (6). However, the radial field (along $x$ ) mixes the $m$ values. This electric field is $\mathrm{E}_{\mathrm{rad}}(r)=e n_{p} r /\left(2 \epsilon_{0}\right)$ for $r \leqslant r_{p}$ and $\mathrm{E}_{\mathrm{rad}}(r)=e n_{p} r_{p}^{2} /\left(2 r \epsilon_{0}\right)$ for $r>r_{p}$ (it reaches $10^{3} \mathrm{~V} / \mathrm{m}$ at the plasma radius $r_{p}=1 \mathrm{~mm}$ for a density $\sim 10^{14} \mathrm{~m}^{-3}$ ). This implies that for a Pn with a radial velocity component of $v_{t h}=912 \mathrm{~m} / \mathrm{s}$ created at the center of a plasma with density $n_{p}=10^{14} \mathrm{~m}^{-3}$ and $r_{p}=1 \mathrm{~mm}, \mathbf{E}$ reaches $\sim 4 \times 10^{3} \mathrm{~V} / \mathrm{m}$ per $\mu$ s of flight. During the time this Pn reaches the plasma edge at $r_{p}$, the sum of electric fields caused $N_{S t, x}=\frac{r_{p}}{v_{t h}} v_{S t, x} \approx 10^{4}$ Stark transitions. Assuming a Rydberg state of $\left\langle n_{P n}\right\rangle \sim 1300$ this transit then yields a mean survivability until annihilation occurs of $\sim 15 \%$, when using the same model as for Fig. 2(f).

(3) The motional Stark effect is a very similar effect because the Pn velocity component perpendicular to $\mathbf{B}$ gives a Lorentz electric field, reaching values of $\mathrm{E}_{m}=4.5 \times 10^{3}$ $\mathrm{V} / \mathrm{m}$ for a Pn moving in radial direction at $v_{t h}=912 \mathrm{~m} / \mathrm{s}$.

These fields of $\mathrm{kV} / \mathrm{m}$ are important, and in addition to Stark quenching they may cause field ionization of the Pn highly excited states. For instance, in axial plasma direction the electric potential rises within a few Debye lengths typically of $\sim 20 \mu \mathrm{m}$ to values of the applied axial trapping potential. For the considered trap geometries and densities the potential change over this short distance leads to peak values of up to $\mathrm{E}_{a x} \approx 3 \times 10^{3} \mathrm{~V} / \mathrm{m}$ on axis at the plasma edges $[24,62,63]$. A polarized Rydberg Pn moving parallel to $B$ ionizes when the electric field surpasses the value in Pn atomic units of $E_{\max }>1 /\left(16 n_{\mathrm{Pn}}^{4}\right)$ [64-66]. With the Pn atomic units of the electric field of $4 \times 10^{17} \mathrm{~V} / \mathrm{m}$ we find for $\mathrm{E}_{a x}$ about $n_{\mathrm{Pn} \text {,ion }}^{a x} \approx 1700$. Thus for $\mathrm{Pn}$ formation from $n_{\mathrm{H}}=30$ a fraction of $\eta_{\text {ion, } 30}=0.84$ of the Pn are formed below the threshold $n_{\mathrm{Pn}, \text { ion }}^{a x}\left[n_{\mathrm{Pn}}\right.$ distribution; see Fig. 2(a)].

From all this discussion and limitations, we see that, if most of the Pn should survive, the Stark mixing and the field ionization (especially at the plasma edges) as Pn travel through the trap has to be considered carefully to give the proper Pn formation number. This is why we have performed a more detailed Monte Carlo simulation to estimate the expected Pn production yield.

We mention that this simulation is based on the simple assumption that the Pn motion through the trap is an undeflected trajectory. This is a reasonable assumption because the transition into "guiding center atoms" where the magnetron motion causes the bound systems to drift apart occurs for $n_{\mathrm{Pn}, \text { ion }}^{\mathrm{rad}} \approx 11000$ [64,65,67-69].

\section{Pn YIELD SIMULATION}

In order to confirm the large production number estimated in Sec. II, we studied in more detail the Pn formation and annihilation process using a Monte Carlo simulation. Here we analyzed the Pn yield into $4 \pi, N_{\mathrm{Pn}}^{4 \pi}$ and into the forward solid 
angle of $\Omega=30 \mathrm{msr}, N_{\mathrm{Pn}}^{\Omega}$, for various plasma parameters and including Stark effects using random positioning $\mathbf{r}_{i}$ for $\mathrm{H}^{-}$ and a 3D Maxwellian distribution for the mixed plasma in thermal equilibrium. In azimuthal direction for each $i$ th $\mathrm{H}^{-}$, the plasma rotation velocity, $v_{\phi}\left(r_{i}\right)=r_{i}$ e $n_{p} /\left(2 \epsilon_{0} B\right)$, is added at the respective particle's radial position $r_{i}$. The number of $\mathrm{H}(30 d)$ are randomly taken determined by $\eta_{L}$. We then use a Metropolis-Hastings method to choose the Pn formation according to the cross section from Eq. (2) and with the $n_{\mathrm{Pn}}$ and $l_{P n}$ state distributions from Fig. 2.

After the laser interaction at $t_{0}$, during each time step of typically $d t=10 \mathrm{~ns}$ for each Pn the sum of $\mathbf{E}_{j}=\mathbf{E}_{m, j}+$ $\mathbf{E}_{\mathrm{rad}, j}+\mathbf{E}_{a x, j}$ defining the quantization axis $z$ is calculated. Between two time steps, a variation of $\mathbf{E}$ defines the perturbation field $\mathbf{E}_{p}$ and determines the number of Stark total transitions $N_{S t, j}=d t\left(v_{S t, x, j}+v_{S t, z, j}\right)$ from Eq. (6) that occur. Per $d t, N_{S t, j}$ transitions are then performed for each $\operatorname{Pn}(n, l)$, where the probability for a $\Delta m=+1, \Delta m=-1, \Delta l=+1$ or $\Delta l=-1$ transition is chosen with a Metropolis-Hastings method according to the individual $v_{S t, x, j}$ and $v_{S t, z, j}$ frequencies. Pn that hereby reach $m_{\mathrm{Pn}}=0, \pm 1$ or $l_{\mathrm{Pn}}=0,1$ during this time step are considered lost and are removed from the simulation and are summed to find $N_{S t}^{4 \pi}$, the number of total Stark-quenched Pn. At $100 \mathrm{~K}$, most Pn formation occurs then within few $\mu$ s.

Figure 4 depicts $N_{\mathrm{Pn}}^{4 \pi}$ (left column) and $N_{\mathrm{Pn}}^{\Omega}$ (right column) for different plasma parameters. From Figs. 4(a) and 4(b) one sees the intuitive picture of an increase in Pn yield with lower $T_{p}$ and with higher $n_{p}$ reaching $N_{\mathrm{Pn}}^{4 \pi}=3 \times 10^{4}$ and $N_{\mathrm{Pn}}^{\Omega}=200$ at $50 \mathrm{~K}$ and $n_{p}=10^{15} \mathrm{~m}^{-3}$. The particle ratio inside the plasma is scanned in Figs. 4(c) and 4(d) showing a plateau at $N_{\mathrm{H}^{-}} / N_{\bar{p}} \approx 1-2$ at $n_{p} \leqslant 10^{14} \mathrm{~m}^{-3}$, which is shifted to larger ratios of two to five for higher densities. The flux into $\Omega$ reaches $\sim 100 \mathrm{Pn}$ for $n_{p}=10^{15} \mathrm{~m}^{-3}$ and for a particle ratio of one to five. In Figs. 4(e) and 4(f) the plasma aspect ratio is scanned while keeping $N_{\bar{p}}$ fixed at $3 \times 10^{7}$ for a density of $10^{15} \mathrm{~m}^{-3}$. Here for the case of keeping the laser intensities constant when varying the plasma radii (increasing laser powers with larger radius), a flat yield distribution is seen with the maximum flux into $\Omega$ at around $A_{l / r}=1\left(r_{p}=1 \mathrm{~mm}, l_{p}=\right.$ $100 \mathrm{~mm}$ ). For the case of keeping the laser powers constant and adjusting the laser waists to the respective plasma radius in the calculation (thereby increasing the laser intensities with smaller radii and $\eta_{L}$ ) a plasma with large $A_{l / r}$ (small radius) exhibits a higher Pn yield.

Because per laser shot about $\eta_{L}=30 \%$ of the $\mathrm{H}^{-}$are excited to $\mathrm{H}(30 d)$ and about $10^{4} \bar{p}$ are used to produce $\mathrm{Pn}$, the remaining plasma still carries sufficient constituents to repeat the formation process. The repetition rate is, however, limited by the pulsed laser repetition rate and the rethermalization time of the plasma. Eventually, a large enough number of $\bar{p}$ and $\mathrm{H}^{-}$have been either transformed to $\mathrm{Pn}$, ionized, or have escaped the trap as $\mathrm{H}$, so that it becomes beneficial to recycle the plasma with new particles. For the rethermalization time, the released photoelectrons from $\mathrm{H}^{-}$ photodetachment at kinetic energies of about $0.42 \mathrm{eV}$ (BE$\left.h c / \lambda_{1064}\right)$ will cause additional heating of the plasma, which will be offset by the continuous electron cooling of the initially trapped electrons. For example, at parameters as used in Fig. 4 of $n_{p}=5 \times 10^{14} \mathrm{~m}^{-3}, N_{H^{-}} / N_{\bar{p}}=1$, and $N_{e^{-}} /\left(N_{H^{-}}+\right.$
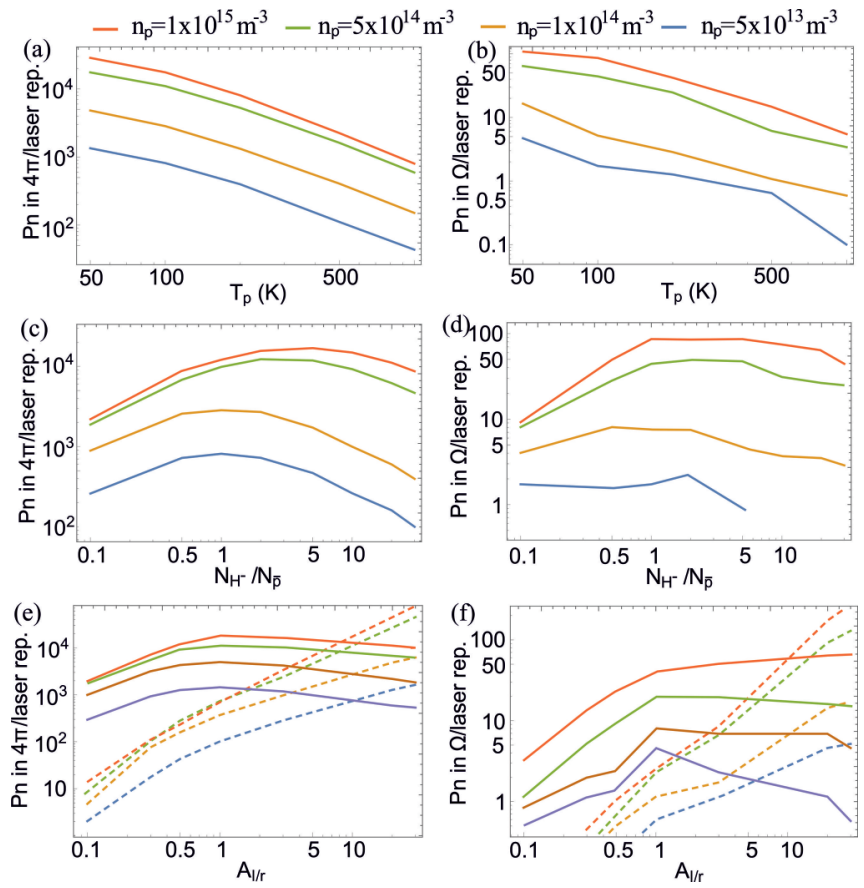

FIG. 4. Number of formed Pn in $4 \pi$ (left column) and into $\Omega$ (right column) for one laser shot for the following plasma parameters: $T_{p}=100 \mathrm{~K}, r_{p}=1 \mathrm{~mm}, l_{p}=100 \mathrm{~mm}, N_{\mathrm{H}^{-}} / N_{\bar{p}}=2, B=5 \mathrm{~T}$, $n_{\mathrm{H}}=30$ for densities $n_{p}=5 \times 10^{13}$ to $10^{15} \mathrm{~m}^{-3}$. While leaving the other parameters constant, in panels (a) and (b) the plasma temperature is scanned, in panels (c) and (d) the ratio of the number of $\mathrm{H}^{-}$to $\bar{p}$ and, in panels (e) and (f) the plasma aspect ratio $A_{l / r}$. To keep the plasma volume $\pi r_{p}^{2} l_{p}$ constant, $A_{l / r}$ is defined via $r_{p}=10^{-5 / 2} A_{l / r}^{-1 / 2}$ and $l_{p}=10^{-2} A_{l / r}$, for (solid lines) constant laser intensities and (dashed lines) constant laser powers. See text for details.

$\left.N_{\bar{p}}\right)=0.1$ there are $N_{e^{-}}=9 \times 10^{7}$ electrons and per laser shot $7 \times 10^{6} \mathrm{H}^{-}$are removed and photoelectrons added. For these photoelectrons the time constant for radiative cooling is given by $\tau_{e l}=\frac{3 \pi m_{\epsilon}^{3} \epsilon_{0} c^{3}}{e^{4} B^{2}}=0.1 \mathrm{~s}^{-1}$, and using coupled rate equations, described in Ref. [70], we find that this heats the plasma temperature initially by about $50 \mathrm{~K}$ within $1 \mathrm{~ms}$ before reaching initial temperatures again after about $0.8 \mathrm{~s}$. Thus, in this example an experimental repetition rate of about $1 \mathrm{~Hz}$ is feasible, which rate can easily be reached by typical pulsed laser systems.

The increase of $N_{\mathrm{Pn}}^{4 \pi}$ as a function of the number of consecutive laser repetitions is then plotted in Fig. 5(a) for $n_{\mathrm{H}}=15$ to 45 . As each shot reduces the number of available particles the curves flatten at about 10 repetitions. For this case, Figs. 5(b) and 5(c) show the numbers of accumulated $\mathrm{Pn}$, ionized $\mathrm{H}$ and Pn, and Stark-quenched Pn within $4 \pi$ and $\Omega$, respectively. Here higher $n_{\mathrm{H}}$ occupations result in more Stark losses and ionizations from inelastic collisions, resulting in a flat distribution of the Pn yield from $n_{\mathrm{H}}=20$ to 40 . For $n_{\mathrm{H}}=45$ the Pn yield decreases, and it reaches a maximum into $\Omega$ at $n_{\mathrm{H}}=30$ of $194 \mathrm{Pn}$ after 10 repetitions. This then corresponds to a total of $7 \times 10^{4}$ formed Pn for $\mathrm{H}(n=30)$, and thus $n_{H}=30$ is used for the laser excitation calculation. 

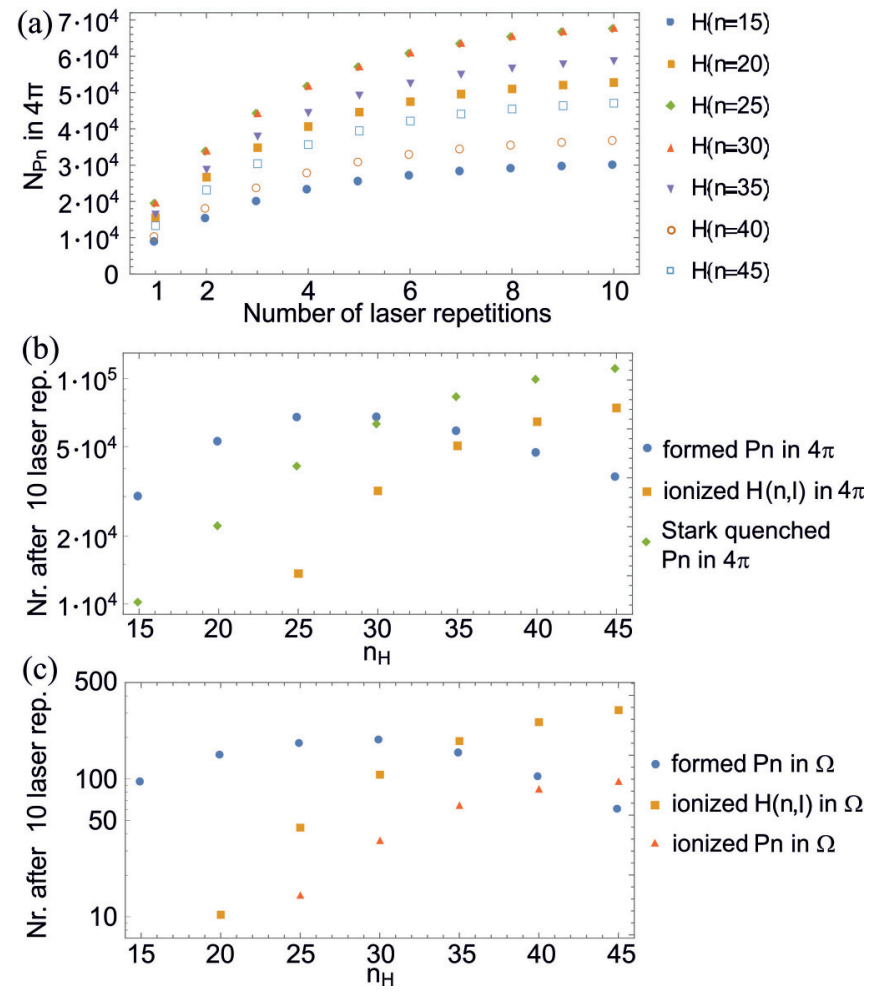

FIG. 5. Number of formed Pn as a function of consecutive laser repetitions and of the initial Rydberg excitation state of $\mathrm{H}$ using the parameters $r_{p}=1 \mathrm{~mm}, l_{p}=100 \mathrm{~mm}, N_{\mathrm{H}^{-}} / N_{\bar{p}}=1, B=5 \mathrm{~T}, n_{p}=$ $5 \times 10^{14} \mathrm{~m}^{-3}$, and $T_{p}=100 \mathrm{~K}$. (a) $N_{\mathrm{Pn}}^{4 \pi}$ versus the number of consecutive laser repetitions for $n_{\mathrm{H}}=15-45$ resulting in $\eta_{m b, 15}=0.99$, $\eta_{m b, 20}=0.95, \eta_{m b, 25}=0.83, \eta_{m b, 30}=0.68, \eta_{m b, 35}=0.53, \eta_{m b, 40}=$ 0.42 , and $\eta_{m b, 45}=0.33$. After 100 laser repetitions: (b) $N_{\mathrm{Pn}}^{4 \pi}, N_{\mathrm{H}, \text { ion }}^{4 \pi}$ and $N_{S t}^{4 \pi}$ versus $n_{\mathrm{H}}$ and (c) $N_{\mathrm{Pn}}^{\Omega}, N_{\mathrm{Pn}, \text { ion }}^{\Omega}$, and $N_{\mathrm{H} \text {,ion }}^{\Omega}$ versus $n_{\mathrm{H}}$. To find $N_{\mathrm{Pn}, \text { ion }}^{\Omega}$, the fractions of Pn with $n<n_{\mathrm{Pn} \text {,ion }}^{a x}$ are $\eta_{\text {ion, } 15}=1, \eta_{\mathrm{ion}, 20}=$ $0.99, \eta_{\text {ion }, 25}=0.93, \eta_{\text {ion }, 30}=0.84, \eta_{\text {ion }, 35}=0.71, \eta_{\text {ion }, 40}=0.55$, and $\eta_{\text {ion }, 45}=0.39$. See text for details.

\section{SUMMARY AND OUTLOOK}

In this paper an ab initio simulation is performed in a Penning-Malmberg trap to produce a pulsed source of highly excited Pn that exhibit long-lived states and with temperatures corresponding to the cryogenic environment in which they are formed. The scheme is based on the preparation of $\bar{p}$ to interact with Rydberg excited $\mathrm{H}(30 d)$ that are produced from photodetached and excited $\mathrm{H}^{-}$. The laser excitation scheme was calculated using a semiclassical picture where in a $5 \mathrm{~T}$ field an excitation efficiency of about 30\% was found for standard laser parameters. The excitation efficiency is taken as an input to perform a Monte Carlo simulation of the interacting $\bar{p}$ and $\mathrm{H}(30 d)$ to produce $\mathrm{Pn}^{*}$ via a resonant-chargeexchange reaction. For a mixed plasma of the constituents at a temperature of $100 \mathrm{~K}$ the Pn formation was found to yield about $7 \times 10^{4} \mathrm{Pn}$ into $4 \pi$ and $\sim 200 \mathrm{Pn}$ into a solid angle of 30 msr towards the Penning trap opening per experimental cycle of $10 \mathrm{~s}$ with a $1 \mathrm{~Hz}$ laser repetition rate.

In an experiment, Pn created from this proposed method could be used to perform spectroscopic studies of Pn Rydberg states, from which the Rydberg constant of Pn can be determined to potentially higher precision than in antiprotonic helium, as the three-body calculation of the later system is simplified to a pure QED two-body calculation. Laser light in the microwave and optical range can drive individual Rydberg transitions, probing the strong force close to the annihilation threshold, where the state detection could be performed by optically pumping the considered state into either a short-lived $s$ and $p$ state or into weakly bound states using field ionization. Also, part of the spontaneously emitted light, which is radiated by $\mathrm{Pn}^{*}$ when cascading down before annihilating from low level states, can be collected and sent to a spectrometer leading to the possibility of an improved (in the case of Pn) or first (in the case of antiprotonic deuterium) measurement of the strong-interaction shift of the ground state.

Indeed, the production scheme can be also extended to antiproton-deuteron atoms, using initially deuterium anions and with the laser excitation frequencies increased by the deuterium level shift of $0.027 \%$ compared to $\mathrm{H}$, and more generally to any atomic system susceptible to be photoionized from its anionic form and excited into a Rydberg state.

Further, the presented Pn source could be used as an input to a Talbot-Lau type interferometer. The Stark effect could be used to create three laser gratings to quench $\mathrm{Pn}$ at the antinodes, resulting in an interference pattern, similar to the ionization laser gratings used in Refs. [71-73]. Such a device could allow performing precision measurements sensitive to the gravitational interaction and to the charge neutrality between $p$ and $\bar{p}$. Here using the scheme of Ref. [11], the phase shift induced by gravity could be measured by horizontally rotating the IF grating plane to control the influence of $g$ during the measurement, i.e., by a rotation of $30^{\circ}$ to be sensitive to $0.5 \mathrm{~g}$. Similarly as discussed for $\bar{H}$ in Ref. [74], Pn at a kinetic energy of $10 \mathrm{meV}$ and for gratings with a periodicity of $1 \mu \mathrm{m}$ and separated by $10 \mathrm{~cm}$ would then allow to resolve $\mathrm{g}$ to a $1 \sigma$ precision after detecting approximately 1000 Pn behind the interferometer. To reach the sensitivity needed to tackle the know neutrality of $\mathrm{Pn}\left(7 \times 10^{-10} \mathrm{C}\right.$ at $90 \%$ confidence interval) [75], in such an IF only a small bias electric field of $\sim 1 \mathrm{~V} / \mathrm{cm}$ perpendicular to the IF axis would be required, while observing an unperturbed interference fringe. Because larger fields can be applied, we think that such Pn source and interferometric measurement is promising for neutrality tests.

\section{ACKNOWLEDGMENT}

We would like to thank C. Drag and Ch. Blondel, Laboratoire de physique des Plasmas-UMR 7648, for valuable discussions and their support working on the simulation.

\section{APPENDIX}

In this Appendix we will start by describing the first- and second-order perturbation theory of two-body bound atoms (Pn, H, and for completeness Ps) in $\mathbf{E}$ and $\mathbf{B}$ fields given in generalized reduced atomic units. For $\mathrm{H}$, the problem is treated in more detail in Refs. [27,76-79].

While such treatments of the overlap of the radial wave function of $\mathrm{H}$ can be found in many works [27,80-82], they are often given only partially or are misprinted. We will then 
recollect the relevant formulas to calculate the light-atom interaction for two-body atoms in a semiclassical picture.

\section{A. Two-body atoms in $\mathbf{E}$ and B fields (perturbation theory)}

In Ref. [27] Eq. (42.1) gives the proper Hamiltonian for two particles in free field, so is valid for $\mathrm{H}, \mathrm{Pn}$, and Ps. To add the external fields we typically change $\mathbf{p}$ to $\mathbf{p}-e \mathbf{A} / c$ where $\mathbf{A}=\frac{1}{2} \mathbf{B} \times \mathbf{r}$ for a uniform magnetic field. Using SI units we find the Hamiltonian in external electric $\mathbf{E}^{\prime}$ and magnetic B fields is given by (where we did not put the relativistic terms such as the spin-orbit ones) [27]: $H=$ $H_{0}+H_{\text {Zeeman }}+H_{\text {Diamagnetic }}+H_{\text {Stark }}$ where $H_{0}=-\frac{\hbar^{2}}{2 \mu} \frac{\partial^{2}}{\partial r^{2}}-$ $\frac{e^{2}}{4 \pi \varepsilon_{0} r}, H_{\mathrm{Zeeman}}^{\mathrm{Pn}}=\mu_{\mathrm{N}} g_{p} \mathbf{B} \cdot \frac{\mathbf{I}_{\mathbf{2}}-\mathbf{I}_{\mathbf{1}}}{2 \hbar}, H_{\mathrm{Zeeman}}^{\mathrm{H}}=\mu_{\mathrm{B}} \mathbf{B} \cdot \frac{-g_{e} \mathbf{S}_{1}+g_{L} \mathbf{L}}{\hbar}-$ $\mu_{\mathrm{N}} \mathbf{B} \cdot \frac{g_{1} \mathbf{I}_{1}}{2 \hbar}, \quad H_{\mathrm{Zeeman}}^{\mathrm{Ps}}=\mu_{\mathrm{B}} g_{e} \mathbf{B} \cdot \frac{\mathbf{S}_{2}-\mathbf{S}_{\mathbf{1}}}{\hbar}$ where $\mu_{\mathrm{B}}=\frac{\hbar e}{2 m_{e}}, \mu_{\mathrm{N}}=$ $\frac{\hbar e}{2 m_{p}}, \mathbf{S}_{\mathbf{1}}$ is the spin operator for the electron, $\mathbf{S}_{\mathbf{2}}$ for the positron, $\mathbf{I}_{\mathbf{1}}$ for the proton and $\mathbf{I}_{\mathbf{2}}$ for the antiproton, and $g_{L} \approx 1-$ $1 / M$, where $M$ is the ratio of the nuclear mass to the electron mass, $g_{e} \approx-2.002319$ and $g_{p} \approx 5.585$. $H_{\text {Diamagnetic }}=$ $-\frac{e^{2}}{8 \mu}(\mathbf{r} \times \mathbf{B})^{2}$, and the Stark effect $H_{\text {Stark }}=-e \mathbf{r} \cdot \mathbf{E}$ has to be calculated using the Lorentz transform to put the Pn, Ps, or $\mathrm{H}$, at rest. So the atom sees a modified electric field $\mathbf{E}=$ $\mathbf{E}^{\prime}+\mathbf{v} \times \mathbf{B}$.

We define reduced units as

(1) The (Bohr) radius $a_{0}^{(\mu)}=\frac{4 \pi \epsilon_{0} \hbar^{2}}{\mu e^{2}}$

(2) The (Hartree) energy $E_{h}^{(\mu)}=\frac{\hbar^{2}}{\mu\left(a_{0}^{(\mu)}\right)^{2}}=\frac{e^{2}}{4 \pi \epsilon_{0} a_{0}^{(\mu)}}$

(3) The reduced electric field $E_{a u}^{(\mu)}=E_{h}^{\mu} /\left(e a_{0}^{(\mu)}\right)$

(4) The reduced magnetic field $B_{a u}^{(\mu)}=\hbar /\left[e\left(a_{0}^{(\mu)}\right)^{2}\right]$.

(5) For $\mathrm{H}$ we have $\mu^{(\mathrm{H})}=m_{e} m_{p} /\left(m_{e}+m_{p}\right) \approx m_{e}$, for Pn $\mu^{(\mathrm{Pn})}=m_{p} / 2 \approx \mu^{(\mathrm{H})} / 914$, and for Ps $\mu^{(\mathrm{Ps})}=m_{e} / 2$. For infinite nucleus mass we have $\mu^{(\infty)}=m_{e}$ and for such case the previous values are the atomic units (values are from Ref. [83]), $E_{h}^{(\infty)}=4.359744722 \times 10^{-18} \mathrm{~J}$, $E_{a u}^{(\infty)}=E_{h} /\left(e a_{0}^{(\infty)}\right)=5.142206748 \times 10^{11} \mathrm{~V} / \mathrm{m}$,magnetic field (SI) $B_{a u}^{(\infty)}=\frac{\hbar}{e\left(a_{0}^{(\infty)}\right)^{2}}=2.350517568 \times 10^{5} \mathrm{~T}$.

Using dimensionless units defined by $r=\bar{r} a_{0}^{(\mu)}, H=$ $\bar{H} E_{h}^{(\mu)}, E=-\bar{E} E_{a u}^{(\mu)}$, and $B=\bar{B} B_{a u}^{(\mu)}$ and using the angular momentum $\overline{\mathbf{L}}=\frac{\mathbf{L}}{\hbar}$ with eigenvalues $m$ on the field-free $|n l m\rangle$ basis and the spin angular momenta $\overline{\mathbf{S}}=\frac{\mathbf{S}}{\hbar}$ and $\overline{\mathbf{I}}=\frac{\mathbf{I}}{\hbar}$ we find

$$
\begin{aligned}
\bar{H}= & -\frac{1}{2} \frac{\partial^{2}}{\partial \bar{r}^{2}}-\frac{1}{\bar{r}}+\overline{\mathbf{r}} \cdot \overline{\mathbf{E}}-\frac{1}{8}(\overline{\mathbf{r}} \times \overline{\mathbf{B}})^{2}+\bar{H}_{\text {Zeeman }}^{(\mu)}, \\
\bar{H}_{\text {Zeeman }}^{(H)}= & \frac{1}{2} \overline{\mathbf{B}} \cdot\left(g_{L} \overline{\mathbf{L}}-g_{e} \overline{\mathbf{S}}_{\mathbf{1}}-\frac{m_{e}}{2 m_{p}} g_{p} \overline{\mathbf{I}}_{\mathbf{1}}\right), \\
\bar{H}_{\text {Zeeman }}^{(P n)} & =\frac{1}{8} g_{p} \overline{\mathbf{B}} \cdot\left(\overline{\mathbf{I}}_{\mathbf{2}}-\overline{\mathbf{I}}_{\mathbf{1}}\right), \\
\bar{H}_{\text {Zeeman }}^{(P s)} & =\frac{1}{4} g_{e} \overline{\mathbf{B}} \cdot\left(\overline{\mathbf{S}}_{\mathbf{2}}-\overline{\mathbf{S}}_{\mathbf{1}}\right),
\end{aligned}
$$

where the magnetic terms differ for $\mathrm{H}, \mathrm{Pn}$, and Ps.

\section{B. Perturbation theory}

The first part of the Hamiltonian $-\frac{1}{2} \frac{\partial^{2}}{\partial \bar{r}^{2}}-\frac{1}{\bar{r}}$ leads to the standard energy levels $-\frac{1}{2 n^{2}}$. If we restrict ourself to a given $n$ manifold we obtain to the following Hamiltonian:

$$
\begin{aligned}
\bar{H} & =-\frac{1}{2 n^{2}}+\overline{\mathbf{r}} \cdot \overline{\mathbf{E}}-\frac{1}{8}(\overline{\mathbf{r}} \times \overline{\mathbf{B}})^{2}+\bar{H}_{\mathrm{B}, \mathrm{L}}^{(\mu)}, \\
\bar{H}_{\mathrm{B}, \mathrm{L}}^{(H)} & =\frac{1}{2} g_{L} \overline{\mathbf{B}} \cdot \overline{\mathbf{L}},
\end{aligned}
$$

where the Zeeman effect of Pn and Ps is independent of $\mathbf{L}$. This Hamiltonian has been studied in several articles, with the pioneering work performed by Pauli, who shows that on the $n$ submanifold $\overline{\mathbf{r}}=-\frac{3}{2} \overline{\mathbf{A}}$, where $\mathbf{A}$ is the Runge-Lenz vector. In this manifold we can define new commuting angular momenta $\mathbf{K}_{1}=\frac{\overline{\mathbf{L}}+\overline{\mathbf{A}}}{2}$ and $\mathbf{K}_{2}=\frac{\overline{\mathbf{L}}-\overline{\mathbf{A}}}{2}$. We shall use the $\left|K_{1} m_{1}\right\rangle \otimes\left|K_{2} m_{2}\right\rangle$ basis where their eigenvalues $m_{1}, m_{2}$ (on a given axis) take the values $-(n-1) / 2,-(n-3) / 2, \ldots,(n-1) / 2$.

\section{First-order perturbation in fields}

Our goal is to calculate the energy levels using perturbation theory. A perturbation theory in field values is when Stark or Zeeman effects are bigger than the fine structure, which is therefore well suited for Rydberg states.

In the uncoupled Paschen-Back basis of $\left|n, l, m_{l}, s, m_{s}, i, m_{i}\right\rangle$ we will focus on the nontrivial orbital part $|n, l, m\rangle$ (notation $m=m_{l}$ ). To the first order in field we thus have to deal with the perturbation for $\mathrm{H}$ as being approximately $\left(g_{L}=1\right.$ and no hyperfine structure) $V_{1}=\overline{\mathbf{r}} \cdot \overline{\mathbf{E}}+\frac{1}{2} \overline{\mathbf{B}} \cdot \overline{\mathbf{L}}$ and for $\mathrm{Pn}$ and $\mathrm{Ps}$ as $V_{1}=\overline{\mathbf{r}} \cdot \overline{\mathbf{E}}$. To stay closer to the $\mathrm{H}$ case and to prepare the second-order perturbation theory we will use the $\left|K_{1} m_{1}\right\rangle \otimes\left|K_{2} m_{2}\right\rangle$ basis. We define for $\mathrm{H} \boldsymbol{\omega}_{1}=\frac{\overline{\mathbf{B}}-3 n \overline{\mathbf{E}}}{2}$ and $\boldsymbol{\omega}_{2}=\frac{\overline{\mathbf{B}}+3 n \overline{\mathbf{E}}}{2}$ and for Ps and Pn $\boldsymbol{\omega}_{1}=-\frac{3 n \overline{\mathbf{E}}}{2}$ and $\boldsymbol{\omega}_{2}=\frac{3 n \overline{\mathbf{E}}}{2}$. In the $n$ manifold, we get $V_{1}=\omega_{1} \cdot \mathbf{K}_{1}+\omega_{2} \cdot \mathbf{K}_{2}$, which can be diagonalized using the $\left|K_{1} m_{1}\right\rangle_{\omega_{1}}\left|K_{2} m_{2}\right\rangle_{\omega_{2}}$ basis. So $m_{1}$ is the projection of $\mathbf{K}_{1}$ on $\omega_{1}$ and $\omega_{1}=\left|\boldsymbol{\omega}_{1}\right|$ and similarly for $m_{2}$. From this the first-order perturbation theory is

$$
\Delta \bar{E}_{m_{1} m_{2}}^{(1)}=\omega_{1} m_{1}+\omega_{2} m_{2} .
$$

For a pure electric field we restore (for $\mathrm{H}$, Ps, and Pn) the Stark effect $\Delta \bar{E}_{m_{1} m 2}^{(1)}=\frac{3}{2} n \bar{E}\left(m_{1}+m_{2}\right)$ with a clear relation to the parabolic quantum number [84]. For $\mathrm{H}$ we restore the standard $\Delta \bar{E}_{m_{1} m 2}^{(1)}=\frac{1}{2} \bar{B}\left(m_{1}+m_{2}\right)$ where $m=m_{1}+m_{2}$ because $\mathbf{K}_{1}+$ $\mathbf{K}_{2}=\overline{\mathbf{L}}$.

The connection with the parabolic eigenfunctions $|n, k, m\rangle$ is $n=n_{1}+n_{2}+|m|+1$ with $n_{1}, n_{2} \geqslant 0$ such that $k=n_{2}-$ $n_{1}$ is the eigenvalue of $A_{z}$. The introduction of parabolic coordinates simultaneously diagonalizes $L_{z}, K_{1 z}, K_{2 z}$. So we can write

$$
\begin{aligned}
|n, k, m\rangle= & \left|K_{1} m_{1}=\frac{1}{2}(m+k)\right\rangle_{\mathbf{E}} \\
& \times\left|K_{2} m_{2}=\frac{1}{2}(m+k)\right\rangle_{\mathbf{E}} .
\end{aligned}
$$

Another parabolic basis often used is the $|n, k, m\rangle$ linked to $\hat{H}, \hat{A}_{z}, \hat{L}_{z}$ eigenvalues, so $k=n_{1}-n_{2}$. It is important to note that using this formalism the states will always be given in a parabolic basis and not in a spherical basis even in a pure magnetic field, so, for instance, $s$ and $p$ states will be mixed.

\section{Second-order perturbation in fields}

The second-order perturbation in fields is due to the diamagnetic term $V_{2}=-\frac{1}{8}(\overline{\mathbf{r}} \times \overline{\mathbf{B}})^{2}$, and the second-order 
Stark effect $W=\overline{\mathbf{r}} \cdot \overline{\mathbf{E}} G_{n} \overline{\mathbf{r}} \cdot \overline{\mathbf{E}}$, with $G_{n}$ is the Green's function of the field-free Hamiltonian. Solov'ev [76] found expressions in the $n$ manifold valid for Pn, Ps, and $\mathrm{H}$ atoms: $V_{2}=\frac{n^{2} \bar{B}^{2}}{16}\left[4 \overline{\mathbf{A}}^{2}-5\left(\overline{\mathbf{A}} \cdot \mathbf{e}_{B}\right)^{2}+\left(\overline{\mathbf{L}} \cdot \mathbf{e}_{B}\right)^{2}+n^{2}+3\right]$ and $W=\frac{n^{4} \bar{E}^{2}}{16}\left[5 n^{2}+31+24 \overline{\mathbf{L}}^{2}-\right.$ $\left.21\left(\overline{\mathbf{L}} \cdot \mathbf{e}_{E}\right)^{2}+9\left(\overline{\mathbf{A}} \cdot \mathbf{e}_{E}\right)^{2}\right]$, where we write the projections on the direction vectors for the electric and magnetic fields as $\mathbf{e}_{E}=\overline{\mathbf{E}} / \bar{E}$ and $\mathbf{e}_{B}=\overline{\mathbf{B}} / \bar{B}$. By taking $\alpha_{1}$ and $\alpha_{2}$ as the angles between the magnetic field $\bar{B}$ and the vectors $\omega_{1}$ and $\omega_{2}$, respectively, (so for Pn and Ps $\left.\alpha_{1}=\pi-\alpha 2\right)$ gives for $\Delta \bar{E}_{m_{1} m 2}^{(2)}=\left\langle n m_{1} m_{2}\left|V_{2}+W\right| n m_{1} m_{2}\right\rangle[76,77]$

$$
\begin{aligned}
\Delta \bar{E}_{m_{1} m_{2}}^{(2)}= & -\frac{n^{4} \bar{E}^{2}}{16}\left[17 n^{2}+19-12\left[m_{1}^{2}+m_{1} m_{2} \cos \left(\alpha_{1}+\alpha_{2}\right)+m_{2}^{2}\right]+\frac{n^{2} \bar{B}^{2}}{48}\left[7 n^{2}+5+4 m_{1} m_{2} \sin \alpha_{1} \sin \alpha_{2}\right.\right. \\
& \left.+\left(n^{2}-1\right)\left(\cos ^{2} \alpha_{1}+\cos ^{2} \alpha_{2}\right)-12\left(m_{1}^{2} \cos ^{2} \alpha_{1}-m_{1} m_{2} \cos \alpha_{1} \cos \alpha_{2}+m_{2}^{2} \cos ^{2} \alpha_{2}\right)\right] .
\end{aligned}
$$

\section{Reduced radial wave function}

For stationary bound states the wave function of a bound two-body system (H, Pn, Ps) with reduced mass $\mu$ can be written in the usual form $\Psi_{n l m}(r, \theta, \phi)=R_{n l}(r) Y_{l m}(\theta, \phi)$ and for continuum states $\Psi_{W l m}(r, \theta, \phi)=R_{W l}(r) Y_{l m}(\theta, \phi)$. Here $W$ labels the free electron kinetic energy linked to an incident photon energy as $\hbar \omega=W+\operatorname{Ry}^{(\mu)} / n^{2}$, where $R y^{(\mu)}=2 E_{h}^{(\mu)}$ is the reduced Rydberg energy. The overlaps of the dipole matrix elements of states $|n, l\rangle$ to $\left|n^{\prime}, l^{\prime}\right\rangle$ can be solved for bound-bound transitions as $R_{n l}^{n^{\prime} l^{\prime}}=\left\langle n^{\prime} l^{\prime}|r| n l\right\rangle=\int_{0}^{\infty} R_{n^{\prime} l^{\prime}}^{*} r R_{n l} r^{2} d r$ and for bound-continuum transitions as $R_{n l}^{W l^{\prime}}=\left\langle W l^{\prime}|r| n l\right\rangle=\int_{0}^{\infty} R_{W l^{\prime}}^{*} r R_{n l} r^{2} d r$. The space-normalized radial wave functions of bound states $R_{n l}$ and of continuum states $R_{W l}$ for a reduced Bohr radius $a_{0}^{(\mu)}$ are then given by

$$
R_{n l}(r)=\sqrt{\frac{(n-l-1) !}{2 n(n+l) !}\left(\frac{2}{n a_{0}^{(\mu)}}\right)^{3}}\left(\frac{2 r^{2}}{n a_{0}^{(\mu)}}\right)^{l} \exp \left(-\frac{r}{n a_{0}^{(\mu)}}\right) L\left(n-l-1 ; 2 l+1 ; \frac{2 r}{n a_{0}^{(\mu)}}\right)
$$

and

$$
\begin{aligned}
R_{W l}(r)= & \sqrt{\frac{2 \Pi_{i=0}^{l}\left(1+i^{2} W / \mathrm{Ry}^{(\mu)}\right)}{\left(1-\exp \left[-\pi \sqrt{4 \mathrm{Ry}^{(\mu)} / W}\right)\right]\left(a_{0}^{(\mu)}\right)^{3} \mathrm{Ry}^{(\mu)}}} \frac{\left(2 r / a_{0}^{(\mu)}\right)^{l}}{(2 l+1) !} \exp \left(i \frac{r}{a_{o}^{(\mu)}} \sqrt{\frac{W}{\mathrm{Ry}^{(\mu)}}}\right) \\
& \times{ }_{1} F_{1}\left(l+1-i \sqrt{\frac{W}{\mathrm{Ry}^{(\mu)}}} ; 2 l+2 ;-2 i \frac{r}{a_{0}^{(\mu)}} \sqrt{\frac{W}{\mathrm{Ry}^{(\mu)}}}\right),
\end{aligned}
$$

with $L$ the generalized Laguerre polynomial and ${ }_{1} F_{1}$ the confluent hypergeometric function. For the bound states the normalization $1=\int_{0}^{\infty} R_{n l}(r)^{2} r^{2} d r$ is used, and for the continuum states we use the energy normalization through $W=\left(k^{(\mu)}\right)^{2} \mathrm{Ry}^{(\mu)}$ and $\delta\left(W-W^{\prime}\right)=\int_{0}^{\infty} R_{W l}(r) R_{W^{\prime} l} r^{2} d r$ so that $R_{k l}(r)=\sqrt{\mathrm{R} y^{(\mu)}} R_{W l}(r)$. To solve the overlap integral for $R_{n l}^{n^{\prime \prime} l^{\prime}}$ an analytical expression for dipole transitions $l^{\prime}=l-1$ is

$$
\begin{aligned}
R_{n l}^{n^{\prime \prime}-1}= & \frac{(-1)^{n^{\prime}-l}}{4(2 l-1) !} \sqrt{\frac{(l+n) !\left(l+n^{\prime}-1\right) !}{(-l+n-1) !\left(n^{\prime}-l\right) !}} \frac{\left(4 n n^{\prime}\right)^{l+1}\left(n-n^{\prime}\right)^{n+n^{\prime}-2 l-2}}{\left(n+n^{\prime}\right)^{n+n^{\prime}}}\left\{{ }_{2} F_{1}\left(l-n+1 ; l-n^{\prime} ; 2 l ;-\frac{4 n n^{\prime}}{\left(n-n^{\prime}\right)^{2}}\right)\right. \\
& \left.-\frac{\left(n-n^{\prime}\right)^{2}}{\left(n^{\prime}+n\right)^{2}}{ }_{2} F_{1}\left[l-n-1, l-n^{\prime} ; 2 l ;-\frac{4 n n^{\prime}}{\left(n-n^{\prime}\right)^{2}}\right]\right\} .
\end{aligned}
$$

$R_{n l}^{n^{\prime} l+1}$ is calculated similarly using $R_{n l}^{n^{\prime} l+1}=R_{n^{\prime} l+1}^{n l}$. The overlap for bound-continuum transitions $R_{n l}^{k l^{\prime}}$ with $k^{(\mu)}=\sqrt{W / \mathrm{R} y^{(\mu)}}$ is given for the cases of $l^{\prime}=l-1$ and $l^{\prime}=l+1$ by

$$
\begin{aligned}
R_{n l}^{k^{(\mu)} l-1}= & \frac{-1}{4(2 l+1) !} \sqrt{\left.\frac{(n+l) ! \Pi_{i=1}^{l-1}\left[1+i^{2}\left(k^{(\mu)}\right)^{2}\right]}{2(n-l-1) !\left[1-\exp \left(2 \pi / k^{(\mu)}\right)\right.}\right]}\left[\frac{4 n}{1+n^{2}\left(k^{(\mu)}\right)^{2}}\right]^{l+1} \exp \left[\frac{-2 \arctan \left(n k^{(\mu)}\right)}{k^{(\mu)}}\right]\left[\frac{n-i / k^{(\mu)}}{n+i / k^{(\mu)}}\right]^{n-l-1} \\
& \times\left\{{ }_{2} F_{1}\left[l-i / k^{(\mu)} ; l+1-n ; 2 l ; \frac{-4 n i / k^{(\mu)}}{\left(n-i / k^{(\mu)}\right)^{2}}\right]-\frac{\left(n-i / k^{(\mu)}\right)^{2}}{\left(n^{\prime}+i / k^{(\mu)}\right)^{2}}{ }_{2} F_{1}\left(l-i / k^{(\mu)} ; l-1-n ; 2 l ; \frac{-4 n i / k^{(\mu)}}{\left(n-i / k^{(\mu)}\right)^{2}}\right)\right\}, \\
R_{n l}^{k^{(\mu)} l+1}= & \frac{-i}{4 k^{(\mu)}(2 l+1) !} \sqrt{\frac{(n+l) ! \Pi_{i=1}^{l-1}\left(1+i^{2}\left(k^{(\mu)}\right)^{2}\right)}{2(n-l-1) !\left[1-\exp \left(2 \pi / k^{(\mu)}\right)\right]}}\left[\frac{4 n}{1+n^{2}\left(k^{(\mu)}\right)^{2}}\right]^{l+2} \exp \left[\frac{-2 \arctan \left(n k^{(\mu)}\right)}{k^{(\mu)}}\right]\left[\frac{n-i / k^{(\mu)}}{n+i / k^{(\mu)}}\right]^{n-l-2} \\
& \times\left\{{ }_{2} F_{1}\left[l+2-i / k^{(\mu)} ; l+1-n ; 2 l+2 ; \frac{-4 n i / k^{(\mu)}}{\left(n-i / k^{(\mu)}\right)^{2}}\right]\right. \\
& \left.-\frac{\left(n-i / k^{(\mu)}\right)^{2}}{\left(n^{\prime}+i / k^{(\mu)}\right)^{2}}{ }_{2} F_{1}\left[l-i / k^{(\mu)} ; l+1-n ; 2 l+2 ;-\frac{4 n i / k^{(\mu)}}{\left(n-i / k^{(\mu)}\right)^{2}}\right]\right\} .
\end{aligned}
$$


To include the fine splitting in the regime of strong fields, where the state is expressed in the uncoupled basis $\left|n l m_{l} s m_{s}\right\rangle$ (for $\mathrm{H}$ in the Paschen-Back regime of strong magnetic fields), the dipole transition between $\left|n l m_{l} s m_{s}\right\rangle$ and $\left|n^{\prime} l^{\prime} m_{l}^{\prime} s^{\prime} m_{s}^{\prime}\right\rangle$ (or $\left|k l^{\prime} m_{l}^{\prime} s^{\prime} m_{s}^{\prime}\right\rangle$ for the case of continuum states) can be found using the Wigner-Eckart theorem that links the dipole moment $\vec{d}=e \vec{r}$ with the radial overlap $R_{n l}^{n^{\prime} l^{\prime}}$ (or $R_{n l}^{k l^{\prime}}$ ) and with $s=s^{\prime}$ as

$$
\begin{aligned}
& \left\langle n^{\prime} l^{\prime} m_{l}^{\prime} m_{s}^{\prime}\left|r^{(q)}\right| n l m_{l} m_{s}\right\rangle \\
& =\delta_{m_{s} m_{s}^{\prime}} C_{l m_{l}, 1\left(q=m_{l}^{\prime}-m_{l}\right)}^{l^{\prime} m_{l}^{\prime}} \frac{\left\langle n^{\prime} l^{\prime} \| r^{\left(q=m_{l}^{\prime}-m_{l}\right)}|| n l\right\rangle}{\sqrt{2 l^{\prime}+1}} \\
& =\delta_{m_{s} m_{s}^{\prime}} C_{l m_{l}, 1\left(m_{l}^{\prime}-m_{l}\right)}^{l^{\prime} m_{l}^{\prime}} C_{l 0,10}^{l^{\prime} 0} \frac{\sqrt{2 l+1}}{\sqrt{2 l^{\prime}+1}} R_{n l}^{n^{\prime} l^{\prime}},
\end{aligned}
$$

where we see directly the selection rule $\Delta m_{s}=0$.

\section{Stimulated emission and photoionization cross section}

The bound-bound cross section between the $\left|n l m_{l} m_{s}\right\rangle$ and $\left|n^{\prime} l^{\prime} m_{l}^{\prime} m_{s}^{\prime}\right\rangle$ states with a energy difference $\hbar \omega$ is given by

$$
\begin{aligned}
\sigma_{n l m_{l} m_{s}}^{n^{\prime} l^{\prime} m_{l}^{\prime} m_{s}^{\prime}}= & \delta_{m_{s} m_{s}^{\prime}} \frac{\pi \omega e^{2}\left(a_{0}^{(\mu)}\right)^{2}}{3 c \epsilon_{0} \hbar} g\left(\omega-\omega_{0}\right) \\
& \times\left|\left\langle n^{\prime} l^{\prime} m_{l}^{\prime}\left|r^{\left(q=m_{l}^{\prime}-m_{l}\right)}\right| n l m_{l}\right\rangle\right|^{2} .
\end{aligned}
$$

Here $g\left(\omega-\omega_{0}\right)$ is the line shape function of the transition with normalization $\int g d \omega=1$ and describes the frequency dependency of the cross section over the ensemble-average line shape. The Einstein $A$ coefficient can then be expressed as function of the bound-bound cross section:

$$
\begin{aligned}
A_{n l m_{l} m_{s}}^{n^{\prime} l^{\prime} m_{l}^{\prime} m_{s}^{\prime}}= & \delta_{m_{s} m_{s}^{\prime}} \frac{\omega^{3} e^{2}\left(a_{0}^{(\mu)}\right)^{2}}{3 \pi \epsilon_{0} \hbar c^{3}} \\
& \times\left|\left\langle n^{\prime} l^{\prime} m_{l}^{\prime}\left|r^{\left(q=m_{l}^{\prime}-m_{l}\right)}\right| n l m_{l}\right\rangle\right|^{2} .
\end{aligned}
$$

Similarly, the cross section for a bound-continuum transition for incident light at angular frequency $\omega$ is

$$
\begin{aligned}
\sigma_{n l m_{l} m_{s}}^{k l^{\prime} m_{l}^{\prime} m_{s}^{\prime}}= & \delta_{m_{s} m_{s}^{\prime}} \frac{16 \pi^{3} a_{0}^{(\mu)} \mathrm{Ry}^{(\mu)} \omega}{c} \\
& \times\left|\left\langle k l^{\prime} m_{l}^{\prime}\left|r^{\left(q=m_{l}^{\prime}-m_{l}\right)}\right| n l m_{l}\right\rangle\right|^{2} .
\end{aligned}
$$

Using a Gaussian laser spectrum with FWMH laser line width $\Delta v_{L}$ gives the laser electric field $E_{L}(t)=\sqrt{2 I(t) /\left(\epsilon_{0} c\right)}$, where $I(t)=\int d \omega I(\omega, t)$ is the entire laser irradiance and $I(t)=I_{0} \exp \left[-4 \ln 2(t / \tau)^{2}\right]$ is the temporal profile with the peak value $I_{0}$ and pulse width $\tau=0.44 / \Delta v_{L}$. For broadband lasers $\left(\Delta \nu_{L} \gg A\right)$ the bound-bound dipole transition rate at resonance is then given for a dipole $d=d_{n l m_{l} m_{s}}^{n^{\prime} l^{\prime} m_{l}^{\prime} m_{s}^{\prime}}=$ $e a_{0}^{(\mu)}\left\langle n^{\prime} l^{\prime} m_{l}^{\prime}\left|r^{\left(q=m_{l}^{\prime}-m_{l}\right)}\right| n l m_{l}\right\rangle$ and a laser polarization $\epsilon$ by

$$
\Gamma_{n l m_{l} m_{s}}^{n^{\prime} l^{\prime} m_{l}^{\prime} m_{s}^{\prime}}(t)=\frac{2 I(t)\left|d \epsilon_{q}\right|^{2}}{\hbar^{2} \epsilon_{0} c \sqrt{\Delta v_{L}^{2}+\Delta v_{D}^{2}}}=\frac{\Omega(t)^{2}}{\sqrt{\Delta v_{L}^{2}+\Delta v_{D}^{2}}},
$$

with the Rabi frequency $\Omega(t)=\boldsymbol{d} \cdot \boldsymbol{E}_{L}(t) / \hbar . \Delta v_{D}$ is the Doppler broadening of the transition for an ensemble of moving atoms at temperature $T$ and particle mass $M$ as $\Delta v_{D}=$ $\omega_{0} / \pi \sqrt{2 k T \ln 2 /\left(M c^{2}\right)}$.

If the photodetachment cross section does not vary significantly over the laser spectral bandwidth (which is the case for the profile of $\mathrm{H}^{-}$and the considered $1064 \mathrm{~nm}$ laser with $\Delta v_{L}=44 \mathrm{MHz}$ ), the photodetachment rate can be written as

$$
\Gamma_{p d}(t)=\frac{I(t)}{\hbar \omega} \sigma_{p d},
$$

with the photodetachment cross section $\sigma_{p d}$. Similarly, the bound-continuum photoionization rate is given by

$$
\Gamma_{n l m_{l} m_{s}}^{k l^{\prime} m_{l}^{\prime} m_{s}^{\prime}}(t)=\frac{I(t)}{\hbar \omega} \sigma_{n l m_{l} m_{s}}^{k l^{\prime} m_{l}^{\prime} m_{s}^{\prime}}
$$

[1] J. Law, Phys. Rev. C 10, 6 (1974).

[2] J. Thaler, J. Thaler, and J. Thaler, J. Phys. G 11, 689 (1985).

[3] D. Gotta, D. Anagnostopoulos, M. Augsburger, G. Borchert, C. Castelli, D. Chatellard, J.-P. Egger, P. El-Khoury, H. Gorke, P. Hauser, P. Indelicato, K. Kirch, S. Lenz, N. Nelms, K. Rashid, T. Siems, and L. Simons, Nucl. Phys. A 660, 283 (1999).

[4] U. Gastaldi and R. Klapisch, Physics at LEAR with Low-Energy Cooled Antiprotons (Springer, New York, 2013).

[5] E. Klempt, F. Bradamante, A. Martin, and J. M. Richard, Phys. Rep. 368, 119 (2002).

[6] E. A. Hessels, D. M. Homan, and M. J. Cavagnero, Phys. Rev. A 57, 1668 (1998).

[7] N. Zurlo, Hyperfine Interact. 172, 97 (2006).

[8] J. S. Cohen and N. T. Padial, Phys. Rev. A 41, 3460 (1990).

[9] R. S. Hayano, Nucl. Phys. A 655, c318 (1999).

[10] E. Lau, Ann. Phys. 437, 417 (1948).

[11] M. K. Oberthaler, S. Bernet, E. M. Rasel, J. Schmiedmayer, and A. Zeilinger, Phys. Rev. A 54, 3165 (1996).

[12] V. A. Kostelecky and A. J. Vargas, Phys. Rev. D 92, 056002 (2015).
[13] V. A. Kostelecky and A. J. Vargas, Phys. Rev. D 98, 036003 (2018)

[14] L. Montanet, Phys. Rep. C 80, 51 (1980).

[15] L. Maiani, A. D. Polosa, and V. Riquer, Phys. Lett. B 750, 37 (2015).

[16] D. R. Entem and F. Fernandez, Phys. Rev. C 73, 045214 (2006).

[17] J. J. Bevelacqua, Phys. Essays 1, 31 (2018).

[18] T. Kalvas, O. Tarvainen, J. Komppula, H. Koivisto, J. Tuunanen, D. Potkins, T. Stewart, and M. P. Dehnel, AIP Conf. Proc. 1655, 030015 (2015).

[19] S. L. Rolston and G. Gabrielse, Hyperfine Interact. 44, 233 (1989).

[20] S. Maury, W. Oelert, W. Bartmann, P. Belochitskii, H. Breuker, F. Butin, C. Carli, T. Eriksson, S. Pasinelli, and G. Tranquille, Hyperfine Interact. 229, 105 (2014).

[21] M. Ahmadi, Nature 541, 506 (2017).

[22] G. Gabrielse, P. Larochelle, D. Le Sage, B. Levitt, W. S. Kolthammer, R. McConnell, P. Richerme, J. Wrubel, A. Speck, M. C. George et al., Phys. Rev. Lett. 100, 113001 (2008).

[23] C. Amsler and A. Ariga, J. Phys.: Conf. Ser. 755, 011001 (2016). 
[24] Y. Enomoto, N. Kuroda, K. Michishio, C. H. Kim, H. Higaki, Y. Nagata, Y. Kanai, H. A. Torii, M. Corradini, M. Leali et al., Phys. Rev. Lett. 105, 243401 (2010).

[25] D. S. Hall and G. Gabrielse, Phys. Rev. Lett. 77, 1962 (1996).

[26] ALPHA Collaboration, G. B. Andresen, M. D. Ashkezari, M. Baquero-Ruiz, W. Bertsche, E. Butler, C. L. Cesar, A. Deller, S. Eriksson, J. Fajans, T. Friesen et al., Nat. Phys. 7, 558 (2011).

[27] H. A. Bethe and E. E. Salpeter, Quantum Mechanics of Oneand Two-Electron Atoms (Springer, Boston, 1957).

[28] G. Gioumousis and D. P. Stevenson, J. Chem. Phys. 29, 294 (1958).

[29] S. Y. Ovchinnikov and J. H. Macek, Phys. Rev. A 71, 052717 (2005).

[30] K. Sakimoto, J. Phys. B 34, 1769 (2001).

[31] J. Cohen, Phys. Rev. A 56, 3583 (1997).

[32] D. L. Morgan and V. W. Hughes, Phys. Rev. D 2, 1389 (1970).

[33] B. D. Esry and H. R. Sadeghpour, Phys. Rev. A 67, 012704 (2003).

[34] K. Sakimoto, Phys. Rev. A 88, 012507 (2013).

[35] K. Sakimoto, Phys. Rev. A 98, 042503 (2018).

[36] K. Sakimoto, Eur. Phys. J. D 72, 17 (2018).

[37] M. Charlton and I. I. Fabrikant, Nat. Commun. 1544, 8 (2017).

[38] D. Krasnický, R. Caravita, C. Canali, and G. Testera, Phys. Rev. A 94, 022714 (2016).

[39] R. Abrines and I. C. Percival, Proc. Phys. Soc. 88, 861 (1966).

[40] R. L. Becker and A. D. MacKellar, J. Opt. Soc. Am. B 17, 3923 (1984).

[41] C. M. Rawlins, A. S. Kadyrov, A. T. Stelbovics, I. Bray, and M. Charlton, Phys. Rev. A 93, 012709 (2016).

[42] B. Zygelman, J. Phys. B 36, L31 (2003).

[43] R. F. Wallis, R. Herman, and H. W. Milnes, J. Mol. Spectrosc. 4, 51 (1960).

[44] J. S. Cohen, Phys. Rev. A 27, 167 (1983).

[45] J. S. Cohen, J. Phys. B: At. Mol. Opt. Phys. 39, 3561 (2006).

[46] K. Sakimoto, Phys. Rev. A 65, 012706 (2001).

[47] B. Y. W. K. Hastings, Biometrika 57, 97 (1970).

[48] M. Rosenbluth and T. Heritage, AIP Conf. Proc. 690, 22 (2003).

[49] J. S. Ball and G. F. Chew, Phys. Rev. 109, 1385 (1958).

[50] B. R. Desai, Phys. Rev. 119, 1385 (1960).

[51] L. P. Yatsenko, V. I. Romanenko, B. W. Shore, T. Halfmann, and K. Bergmann, Phys. Rev. A 71, 033418 (2005).

[52] A. Dalgarno and R. Forrey, J. Phys. B 709, L168 (2010).

[53] H. C. Bryant, B. D. Dieterle, J. Donahue, H. Sharifian, H. Tootoonchi, D. M. Wolfe, P. A. M. Gram, and M. A. YatesWilliams, Phys. Rev. Lett. 38, 228 (1977).

[54] M. Vandevraye, P. Babilotte, C. Drag, and C. Blondel, Phys. Rev. A 90, 013411 (2014).

[55] M. Genevriez and X. Urbain, Phys. Rev. A 91, 033403 (2015).

[56] G. G. Gabrielse, B. G. Lowacz, D. G. Rzonka, C. D. H. Amley, E. A. H. Essels, N. J. Ones, G. K. Hatri, S. A. L. Ee, C. M. Eisenhelder, T. M. Orrison et al., Opt. Lett. 43, 2905 (2018).
[57] A. Messiah, Quantum Mechanics (Dover, New York, 1999).

[58] D. Comparat, Phys. Rev. A 80, 012106 (2009).

[59] D. Comparat, Phys. Rev. Lett. 106, 138902 (2011).

[60] T. B. Day, G. A. Snow, and J. Sucher, Phys. Rev. Lett. 3, 61 (1959).

[61] V. E. Markushin, ITEF 80, 46 (1980).

[62] N. Kuroda, S. Ulmer, D. J. Murtagh, S. Van Gorp, Y. Nagata, M. Diermaier, S. Federmann, M. Leali, C. Malbrunot, V. Mascagna et al., Nat. Commun. 5, 3089 (2014).

[63] G. Gabrielse, N. S. Bowden, P. Oxley, A. Speck, C. H. Storry, J. N. Tan, M. Wessels, D. Grzonka, W. Oelert, G. Schepers et al., Phys. Rev. Lett. 89, 213401 (2002).

[64] D. Vrinceanu, B. E. Granger, R. Parrott, H. R. Sadeghpour, L. Cederbaum, A. Mody, J. Tan, and G. Gabrielse, Phys. Rev. Lett. 92, 133402 (2004).

[65] M. E. Glinsky and T. M. O’Neil, Phys. Fluids B: Plasma Phys. 3, 1279 (1991).

[66] T. F. Gallagher, Rydberg Atoms (Cambridge University Press, New York, 1994).

[67] F. Robicheaux and J. D. Hanson, Phys. Rev. A 69, 010701(R) (2004).

[68] S. G. Kuzmin, T. M. O. N. E. Glinsky, S. G. Kuzmin, and T. M. O. Neil, Phys. Plasmas 11, 2382 (2004).

[69] G. Gabrielse, R. Kalra, W. S. Kolthammer, R. McConnell, P. Richerme, D. Grzonka, W. Oelert, T. Sefzick, M. Zielinski, D. W. Fitzakerley et al., Phys. Rev. Lett. 108, 113002 (2012).

[70] G. Gabrielse, S. L. Rolston, L. Haarsma, and K. Wells, Phys. Lett. A 129, 38 (1988).

[71] S. Nimmrichter, New J. Phys. 13, 075002 (2011).

[72] S. Gerlich, L. Hackermüller, K. Hornberger, A. Stibor, H. Ulbricht, M. Gring, F. Goldfarb, M. Savas, M. Müri, M. Mayor, and M. Arndt, Nat. Phys. 3, 711 (2007).

[73] M. Arndt, N. Dörre, S. Eibenberger, P. Haslinger, J. Rodewald, K. Hornberger, S. Nimmrichter, and M. Mayor, Matter Wave Interferometry with Composite Quantum Objects, Vol. 188 (IOS Press, Amsterdam, 2017).

[74] S. Gerber, J. Phys. B 51, 035008 (2018).

[75] M. Tanabashi, Phys. Rev. D 98, 030001 (2018).

[76] E. A. Solov'ev, Sov. Phys. JETP 58, 63 (1983).

[77] J. Main, M. Schwacke, and G. Wunner, Phys. Rev. A 57, 1149 (1998).

[78] P. A. Braun, Rev. Mod. Phys. 65, 115 (1993).

[79] T. Bartsch, J. Main, and G. Wunner, Phys. Rev. A 67, 063411 (2003)

[80] W. F. Gordon, Ann. Phys. 394, 1031 (1929).

[81] A. Burgess, Mem. Roy. Ast. Soc. 69, 1 (1965).

[82] L. M. Ugray, Heavy Rydberg Photo-dissociation Cross-section Calculations and Experimental Progress Towards Cold Collisions in Lithium (2013), arXiv:1310.5137.

[83] https://physics.nist.gov/cuu/Constants.

[84] Y. N. Demkov, B. S. Monozon, and V. N. Ostrovskii, Zh. Eksp. Teor. Fiz. 57, 1431 (1969) [Sov. Phys. JETP 30, 775 (1970)]. 